\title{
EGFR regulates macrophage activation and function in bacterial infection
}

\author{
Dana M. Hardbower, ${ }^{1,2}$ Kshipra Singh, ${ }^{2}$ Mohammad Asim, ${ }^{2}$ Thomas G. Verriere, ${ }^{2}$ Danyvid Olivares-Villagómez, ${ }^{1}$ Daniel P. Barry, ${ }^{2}$ \\ Margaret M. Allaman, ${ }^{2}$ M. Kay Washington, ${ }^{1}$ Richard M. Peek Jr., ${ }^{2,3}$ M. Blanca Piazuelo, ${ }^{2}$ and Keith T. Wilson ${ }^{1,2,3,4,5}$ \\ 'Department of Pathology, Microbiology and Immunology, and ²Division of Gastroenterology, Hepatology and Nutrition, Department of Medicine, Vanderbilt University Medical Center, Nashville, \\ Tennessee, USA. ${ }^{3}$ Department of Cancer Biology, Vanderbilt University Medical Center, Nashville, Tennessee, USA. ${ }^{4}$ Center for Mucosal Inflammation and Cancer, Vanderbilt University Medical Center, \\ Nashville, Tennessee, USA. ${ }^{5}$ Veterans Affairs Tennessee Valley Healthcare System, Nashville, Tennessee, USA.
}

\begin{abstract}
EGFR signaling regulates macrophage function, but its role in bacterial infection has not been investigated. Here, we assessed the role of macrophage EGFR signaling during infection with Helicobacter pylori, a bacterial pathogen that causes persistent inflammation and gastric cancer. EGFR was phosphorylated in murine and human macrophages during $\boldsymbol{H}$. pylori infection. In human gastric tissues, elevated levels of phosphorylated ECFR were observed throughout the histologic cascade from gastritis to carcinoma. Deleting Egfr in myeloid cells attenuated gastritis and increased $H$. pylori burden in infected mice. EGFR deficiency also led to a global defect in macrophage activation that was associated with decreased cytokine, chemokine, and NO production. We observed similar alterations in macrophage activation and disease phenotype in the Citrobacter rodentium model of murine infectious colitis. Mechanistically, EGFR signaling activated NF- $\mathrm{KB}$ and MAPK1/3 pathways to induce cytokine production and macrophage activation. Although deletion of Egfr had no effect on DC function, ECFR-deficient macrophages displayed impaired Th1 and Th17 adaptive immune responses to $H$. pylori, which contributed to decreased chronic inflammation in infected mice. Together, these results indicate that EGFR signaling is central to macrophage function in response to enteric bacterial pathogens and is a potential therapeutic target for infection-induced inflammation and associated carcinogenesis.
\end{abstract}

\section{Introduction}

Macrophages represent a dynamic subset of innate immune cells, with functions in immune surveillance, immunity to pathogens, wound healing, antigen presentation, and cytokine and chemokine production (1-3). A critical step in macrophage function is macrophage activation (4). Classically activated, M1 macrophages are generated in response to pathogens and proinflammatory cytokines $(1,2,4,5)$. M1 macrophages mediate the antimicrobial response to pathogens $(1,2,4)$. Alternatively activated, M2 macrophages are generated in response to IL-4 and are associated with wound healing $(2,4-6)$ and promotion of tumorigenesis $(7,8)$. Third, regulatory macrophages (Mregs) are antiinflammatory, secreting high concentrations of IL-10 to counter-regulate the M1 response $(4,9)$.

Many studies have focused on signaling pathways that regu-

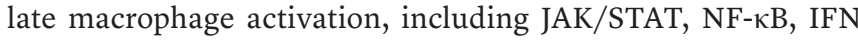
regulatory factor (IRF), and cAMP-responsive element-binding protein (CREB) (10-15). Recent evidence suggests that tyrosine kinase signaling also regulates macrophage activation (16). Specifically, EGFR signaling has been implicated in macrophage function in mouse models of glaucoma, colitis, and cancer (1720). Mice lacking EGFR in resident liver macrophages (Kupffer cells) developed less hepatocellular carcinoma as a direct result

Conflict of interest: The authors have declared that no conflict of interest exists. Submitted: July 6, 2015; Accepted: June 2, 2016.

Reference information: J Clin Invest. 2016;126(9):3296-3312. doi:10.1172/JCI83585. of decreased cytokine production by Kupffer cells (18). However, the role of macrophage EGFR signaling in bacterial infections has not been studied.

To investigate this, we chose a highly prolific human pathogen, Helicobacter pylori (21). H. pylori is a Gram-negative, microaerophilic bacterium that infects approximately $50 \%$ of the global population $(22,23)$. Infection with $H$. pylori is the single greatest risk factor for the development of gastric cancer, which is the third leading cause of cancer death worldwide (24-26). The combination of $H$. pylori-produced oncoproteins, such as cytotoxin-associated gene A (CagA), and infection-induced chronic inflammation, which fails to eradicate the infection, creates a vicious circle of tissue and DNA damage (27-31). Importantly, macrophages play a critical role in $H$. pylori-mediated gastritis (32-34). Thus, an enhanced understanding of the regulation of macrophage responses to $H$. pylori could prove very useful in devising new strategies for chemoprevention of gastric cancer, as antibiotic treatment is often ineffective at eradicating the pathogen, especially in high-risk regions $(28-30,35)$.

To gain further insights into the role of macrophage EGFR signaling in mucosal inflammation driven by bacterial infection, we also used the murine pathogen Citrobacter rodentium, an attaching and effacing enteric bacterial pathogen that mimics enteropathogenic and enterohemorrhagic Escherichia coli (36). C. rodentium infection leads to acute colon inflammation, marked by significant macrophage infiltration $(37,38)$, and has been used as a mouse model of inflammatory bowel disease. 
A
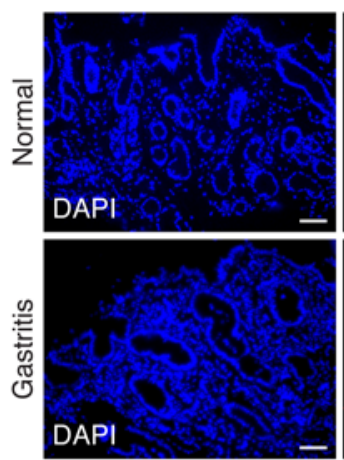
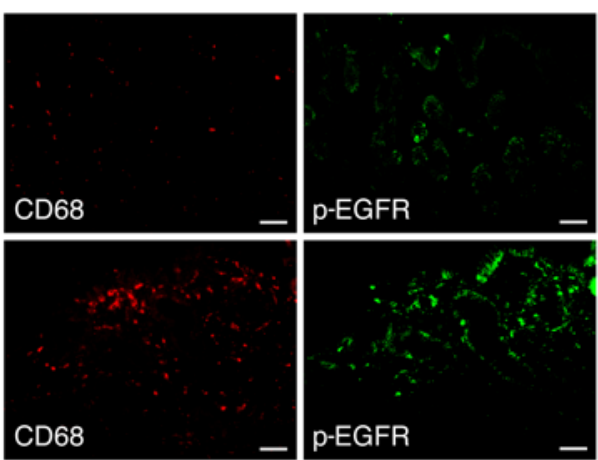
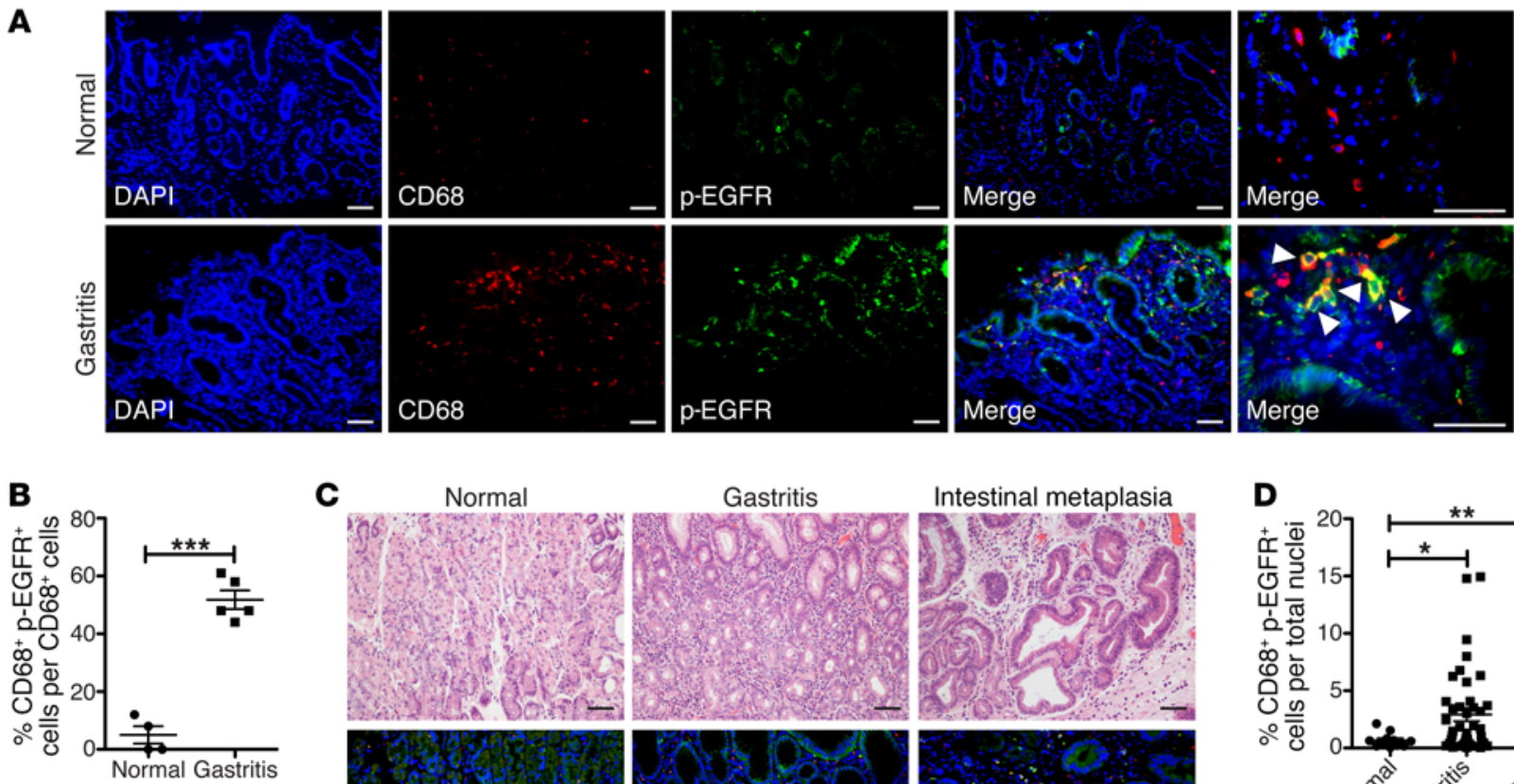

C
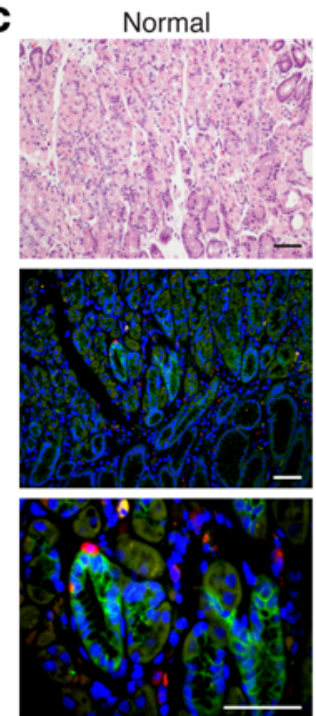

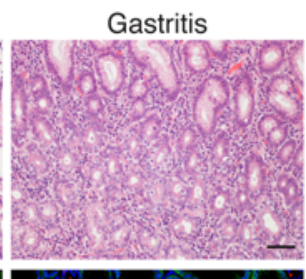

Intestinal metaplasia
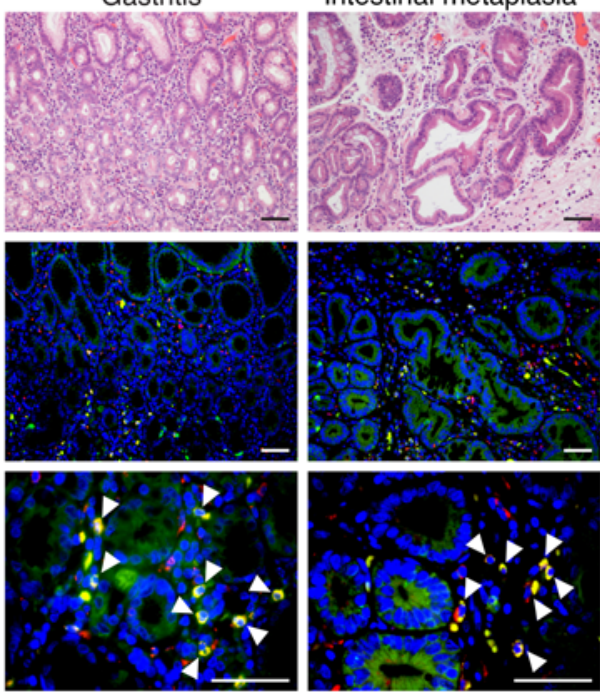

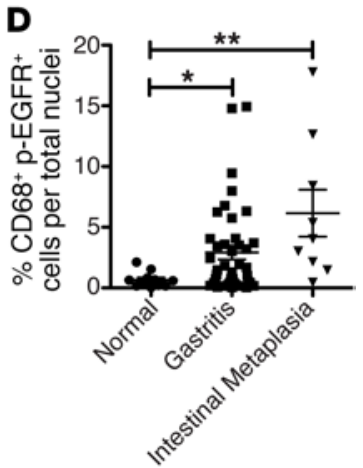

Figure 1. Increased p-EGFR levels in human Gmacs in H. pylori-induced gastritis and precancerous intestinal metaplasia. (A) Representative immunofluorescence images of human gastric biopsies with normal histology and active, nonatrophic gastritis. CD68 = red, EGFR $\mathrm{p}-\mathrm{Y} 1068=$ green; merge = yellow; DAPI = blue. $n=4$ normal samples and 5 gastritis samples. Scale bars: $50 \mu \mathrm{M}$. (B) Quantification of the percentage of CD68 ${ }^{+} p^{-E G F R}{ }^{+}$macrophages among the total number of $\mathrm{CD}_{6} 8^{+}$cells in 10 fields per slide from images shown in $\mathbf{A}$. The slides were analyzed in a blinded manner. $n=4$ normal samples and 5 gastritis samples. ${ }^{* *} P<0.001$, by 2 -tailed Student's $t$ test. (C) Representative H\&E-stained images and immunofluorescence images of gastric tissues from the Vanderbilt University TMA. CD68 = red; EGFR p-Y1068 = green; merge = yellow; DAPI = blue. $n=12$ normal samples, 41 gastritis samples, and 9 intestinal metaplasia samples. Scale bars: $100 \mu \mathrm{M}$. (D) Quantification of the percentage of CD68 ${ }^{+} \mathrm{p}^{-E G F R^{+}}$cells among the total number of cells in each individual core in the TMA, as determined by CellProfiler. $n=12$ normal samples, 41 gastritis samples, and 9 intestinal metaplasia samples. ${ }^{*} P<0.05$ and ${ }^{*} P<0.01$, by 1 -way ANOVA with the Kruskal-Wallis test, followed by the Mann-Whitney $U$ test.

Here, we demonstrate that EGFR signaling a) is activated in macrophages in response to the bacterial pathogens $H$.pylori and C. rodentium; b) plays a critical role in macrophage activation and function; and c) is involved in human macrophages during H.pylori-induced disease progression.

\section{Results}

EGFR phosphorylation in macrophages occurs during human $H$. pylori infection. In previous studies, we determined that phosphorylated EGFR (p-EGFR) in epithelial cells is correlated with early stages of gastric carcinogenesis in humans (39). We also observed that mononuclear cells in the lamina propria of stomach tissues contained p-EGFR (Supplemental Figure 1; supplemental material available online with this article; doi:10.1172/ JCI83585DS1). To determine whether p-EGFR localized to macrophages, we used human tissues from Colombia, a region with a high prevalence of gastric cancer $(27,40,41)$. These gastric biop- sies were from patients with $c a g A^{+} H$. pylori infection and chronic, active gastritis. Using CD68 as a macrophage marker, we found high levels of $\mathrm{CD} 68^{+} \mathrm{p}$-EGFR ${ }^{+}$macrophages in gastritis tissues, levels that were significantly increased compared with those detected in uninfected control patients (Figure 1, A and B). These data indicate that p-EGFR is upregulated in human gastric macrophages (Gmacs) during H.pylori-induced inflammation.

To examine these findings in a larger number of cases, we used a human tissue microarray (TMA) (39) from Vanderbilt University Medical Center that included patients' gastric tissues spanning the histological range from normal to gastric cancer. We stained the TMA for CD68 and p-EGFR (Figure 1C) and used CellProfiler cell image analysis software (http://cellprofiler.org/) to quantify $\mathrm{CD} 68^{+} \mathrm{p}-\mathrm{EGFR}^{+}$cells. The percentage of $\mathrm{CD} 68^{+} \mathrm{p}-\mathrm{EGFR}^{+}$ macrophages was significantly increased in gastritis and in intestinal metaplasia (Figure 1D). Further, the percentage of $\mathrm{CD} 68^{+-}$ p-EGFR ${ }^{+}$macrophages was also increased in both intestinal-type 
A
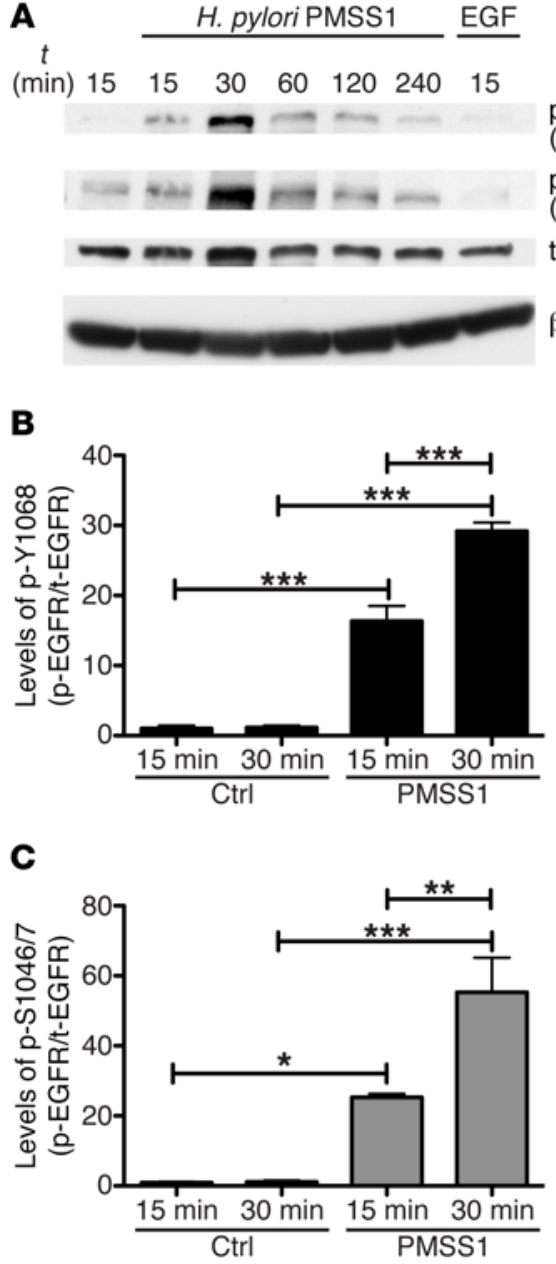

B
$\mathbf{F}$

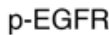

(Y1068)

p-EGFR

(S1046/7)

t-EGFR

$\beta$-Actin

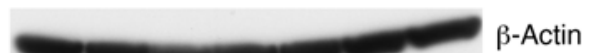

D

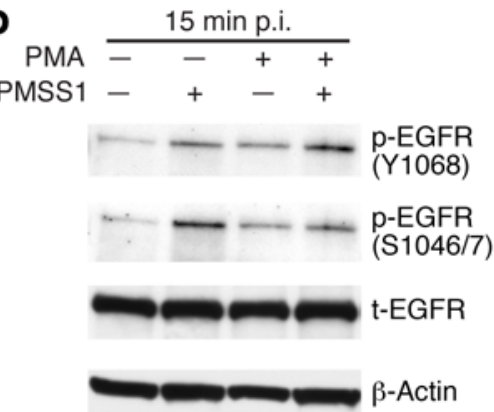

E

PMSS1

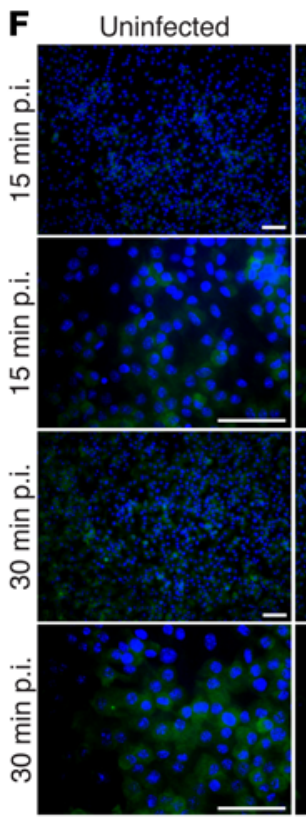

Gefitinib only

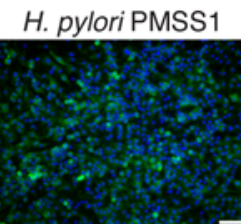

PMSS1 + gefitinib
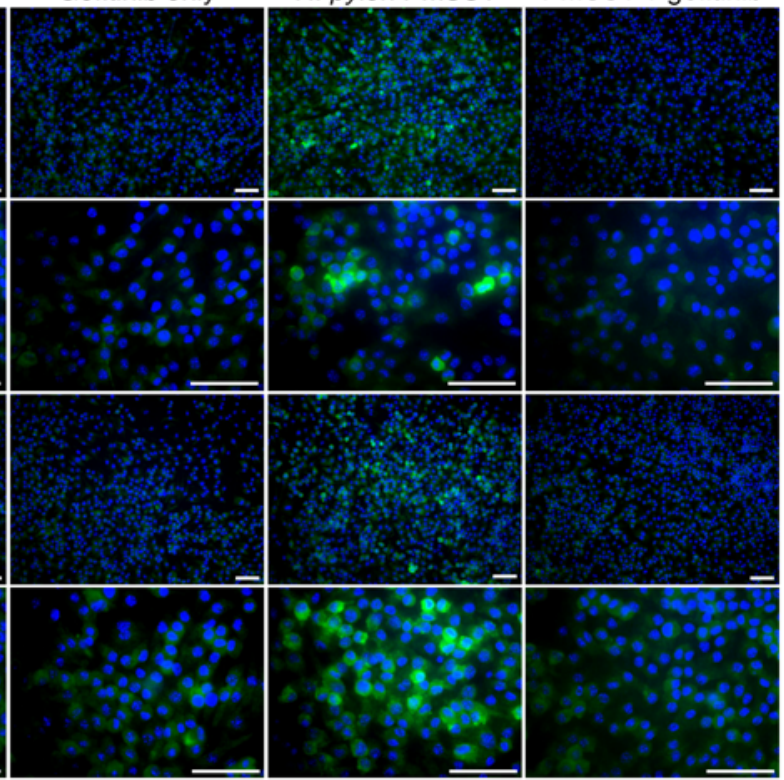

G Untreated

Anti-HB-EGF
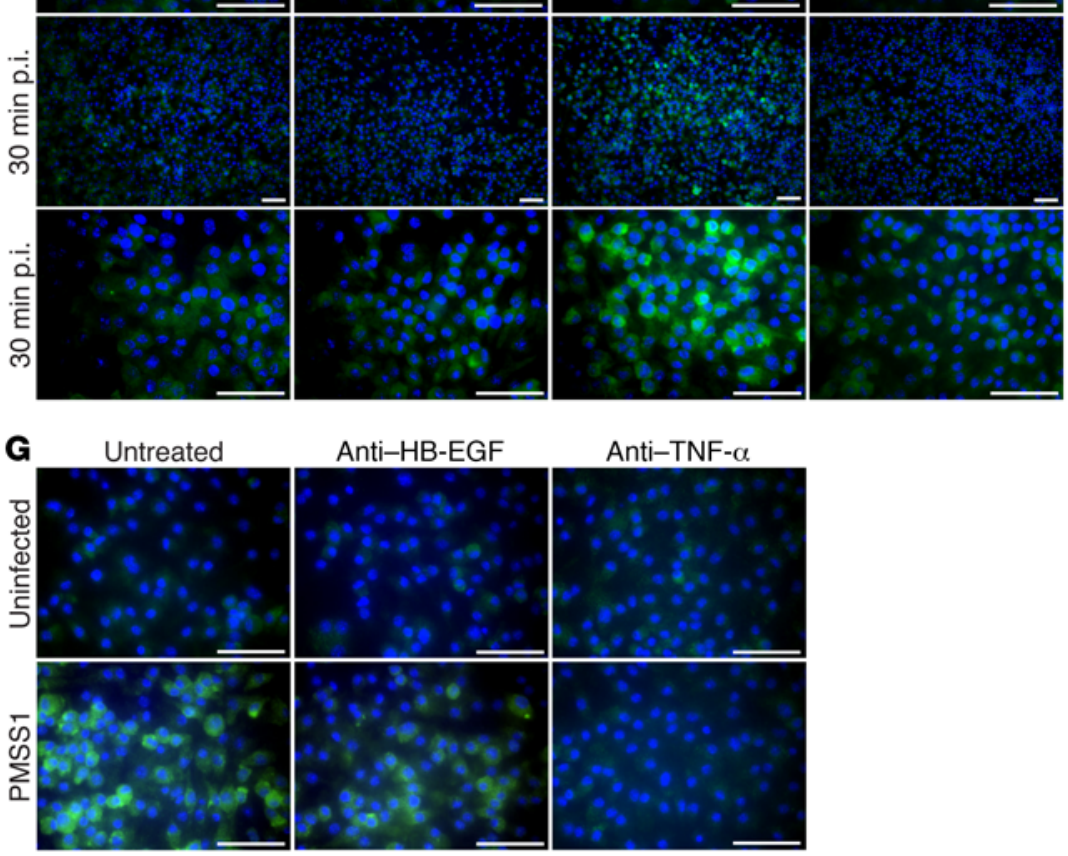

Anti-TNF- $\alpha$

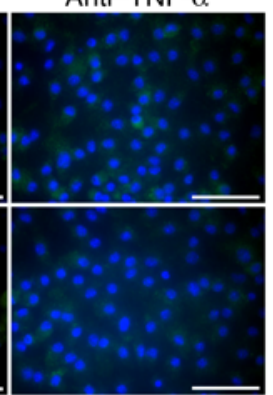

\begin{tabular}{lll}
\multicolumn{3}{r}{15 min p.i. } \\
\hline$-\quad-\quad+$ \\
$-\quad+\quad-\quad+$
\end{tabular}

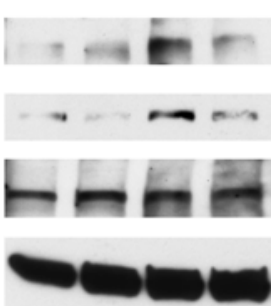

p-EGFR

(Y1068)

p-EGFR

(S1046/7)

t-EGFR

$\beta$-Actin

Figure 2. EGFR signaling in macrophages is induced by $\mathbf{H}$. pylori infection in murine and human macrophages. (A) Representative Western blot of EGFR p-Y1068 and p-S1046/7 in RAW 264.7 cells at various time points p.i. with H. pylori PMSS1 or stimulation with EGF (5 $\mathrm{ng} / \mathrm{ml})$. $n=3$ biological replicates. (B) Densitometric analysis of the levels of $p$-Y1068 compared with levels of t-EGFR at 15 and 30 minutes p.i. $n=3$ biological replicates. ${ }^{* *} P<0.001$, by 1-way ANOVA with Newman-Keuls post test. (C) Densitometric analysis of p-S1046/7 levels compared with t-EGFR levels at 15 and 30 minutes p.i. $n=3$ biological replicates. ${ }^{*} P<0.05$, ${ }^{* *} P<0.01$, and ${ }^{* *} P<0.001$, by 1-way ANOVA with Newman-Keuls post test. (D) Representative Western blot of EGFR at $p-Y 1068$ and $p-S 1046 / 7$ in THP-1 cells at 15 minutes p.i. with H. pylori PMSS1. Monocytes (- PMA) and macrophages (+ PMA) are represented in this blot. $n=3$ biological replicates. (E) Representative Western blot of EGFR at p-Y1068 and p-S1046/7 in RAW 264.7 cells at 15 minutes p.i. with H. pylori PMSS1 $\pm 10 \mu M$ gefitinib. $n=3$ biological replicates. (F) Representative immunofluorescence images of WT BMmacs infected with $\mathrm{H}$. pylori PMSS1 $\pm 10 \mu \mathrm{M}$ gefitinib at the indicated times. EGFR p-Y1068 = green; DAPI = blue. $n=5$ biological replicates. Scale bars: $50 \mu \mathrm{M}$. (C) Representative immunofluorescence images of WT BMmacs infected with $H$. pylori PMSS1 \pm $10 \mathrm{ng} / \mathrm{ml}$ anti-TNF- $\alpha$ or $\pm 25 \mathrm{ng} / \mathrm{ml}$ anti-HB-EGF at 30 minutes p.i. Green = EGFR p-Y1068; blue = DAPI. $n=3$ biological replicates. Scale bars: $50 \mu \mathrm{M}$. Ctrl, control.

and diffuse-type gastric cancer (Supplemental Figure 2, A and B). Importantly, a significant percentage of total $\mathrm{CD} 68^{+}$macrophages was p-EGFR ${ }^{+}$in intestinal metaplasia and in intestinal-type and diffuse-type gastric cancer, indicating that EGFR signaling in mac- rophages has a critical role in gastric carcinogenesis (Supplemental Figure 2C). The percentage of CD68 ${ }^{-} \mathrm{p}-\mathrm{EGFR}^{+}$cells, the majority of which are epithelial cells, decreased in both types of gastric cancer (Supplemental Figure 3A). These data are consistent with 
A

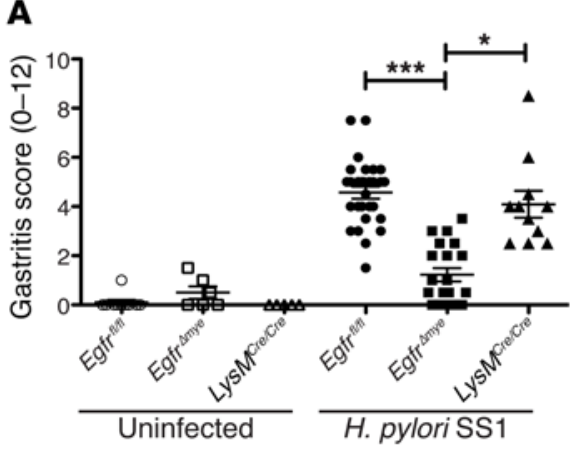

B Egfrivn

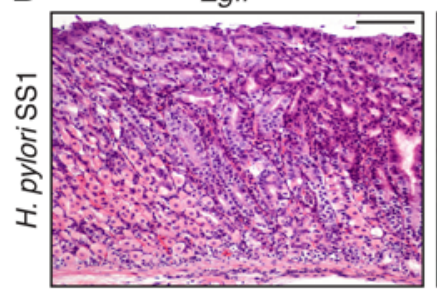

D

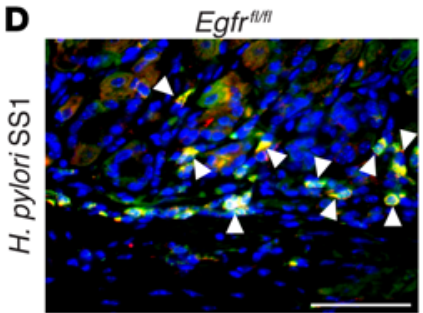

E

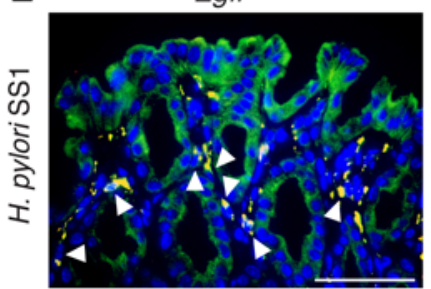

C

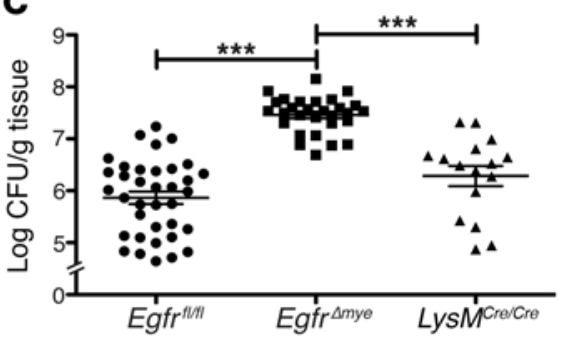

Egfr $\triangle$ mye

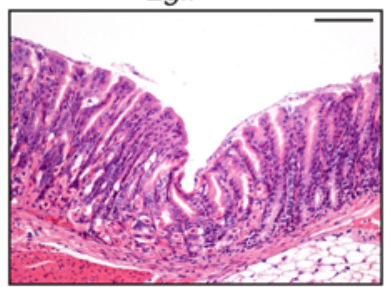

Egfr $\triangle m y e$

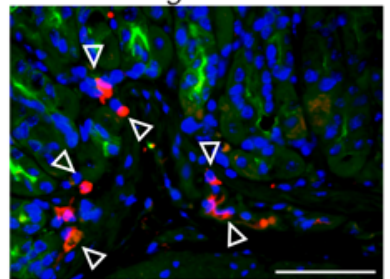

Egframye

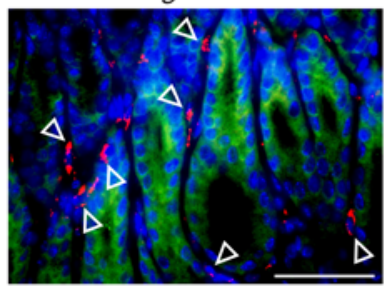

LysM $M^{\text {Cre/Cre }}$

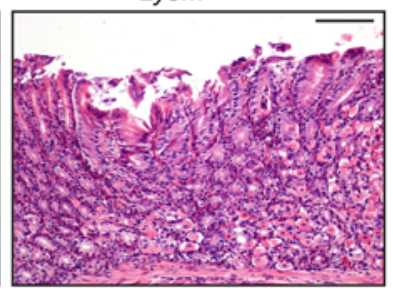

LysMcredcre

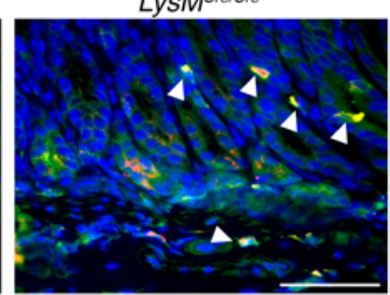

LysMcreacre

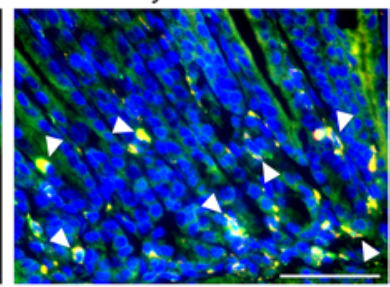

Figure 3. Egfr ${ }^{\text {dmye }}$ mice have significantly increased $\boldsymbol{H}$. pylori burden but significantly decreased gastritis after chronic infection.

(A) Gastritis scores were assessed in a blinded manner by a gastrointestinal pathologist 4 months p.i. according to the updated Sydney System. ${ }^{*} P<0.05$ and ${ }^{* *} P<0.001$, by 1-way ANOVA with Newman-Keuls post test. (B) Representative H\&E-stained images from infected mice in A. Scale bars: $100 \mu \mathrm{M}$.

(C) Colonization of H. pylori SS1 was assessed by serial dilution and culture 4 months p.i. ${ }^{* * *} P<0.01$, by 1 -way ANOVA with NewmanKeuls post test. $n=5-10$ uninfected and 11-30 H. pylori 551 mice per genotype (A and $\mathbf{C}$ ). (D) Representative immunofluorescence images of $p$-EGFR from infected mice in $\mathbf{A}$ and $\mathbf{B}$. EGFR p-Y068 = green; CD68 = red; merge = yellow; DAPI = blue. Solid arrowheads indicate $\mathrm{CD}^{+} 8^{+} \mathrm{p}-\mathrm{EGFR}^{+}$macrophages; open arrowheads indicate $\mathrm{CD}^{+} 8^{+} \mathrm{p}-$ EGFR ${ }^{-}$macrophages. Scale bars: $50 \mu \mathrm{M}$. (E) Immunofluorescence images of t-EGFR from infected mice in $\mathbf{A}$ and $\mathbf{B}$. t-EGFR = green CD68 $=$ red; merge = yellow; DAPI = blue. Solid arrowheads indicate CD68 ${ }^{+}$-EGFR ${ }^{+}$ macrophages; open arrowheads indicate CD68 ${ }^{+}$-EGFR- macrophages. Scale bars: $50 \mu$ M. $n \geq 3$ mice per genotype ( $\mathbf{D}$ and $\mathbf{E}$ ). those from our previous report, in which we demonstrated diminished p-EGFR levels in gastric epithelial cells by immunoperoxidase staining in cases of gastric cancer compared with levels in earlier stages of disease (39). While there was an overall positive correlation between the percentage of $\mathrm{CD}^{+} 8^{+} \mathrm{p}-\mathrm{EGFR}^{+}$and the percentage of $\mathrm{CD}^{-} \mathrm{p}-\mathrm{EGFR}^{+}$cells (Supplemental Figure 3B), this was driven primarily by cases of active gastritis (Supplemental Figure 3C), suggesting a potential interaction between macrophages and non-macrophages in the induction of EGFR signaling during gastric inflammation. Importantly, the correlation between macrophage and non-macrophage/epithelial p-EGFR levels was lost in intestinal-type and diffuse-type gastric cancer (Supplemental Figure 3C), indicating that macrophage EGFR signaling is independent of epithelial EGFR signaling at the end stages of the histologic cascade. These data demonstrate that EGFR signaling plays an important role in macrophage function during chronic inflammation and carcinogenesis in the human stomach.

H. pylori infection induces EGFR phosphorylation in macrophages. To extend these observations, we determined whether H. pylori infection is sufficient to induce EGFR phosphorylation in macrophages in vitro. In murine RAW 264.7 cells, p-EGFR levels peaked at 30 minutes postinfection (p.i.) (Figure 2A). Phosphorylation was detectable at 2 residues, tyrosine (Y) 1068 and serine (S) 1046/7 (Figure 2A). Densitometric analysis confirmed the induction of EGFR phosphorylation at both residues and revealed that the induction at 30 minutes p.i. was significantly greater than the induction at 15 minutes p.i. at p-Y1068 and p-S1046/7 (Figure 2, B and C). Additionally, phosphorylation of EGFR occurred in response to $H$. pylori in the human monocytic cell line THP-1, either with or without differentiation into macrophages (Figure 2D). The clinically available, EGFR-specific tyrosine kinase inhibitor gefitinib effectively diminished EGFR phosphorylation (Figure 2E). Similarly, in primary bone marrow-derived macrophages (BMmacs) from naive $\mathrm{C} 57 \mathrm{BL} / 6 \mathrm{WT}$ mice, $H$. pylori infection was sufficient to induce EGFR phosphorylation, which was attenuated by gefitinib (Figure 2F). Thus, EGFR transactivation is upregulated in macrophages in response to a bacterial pathogen, and it is a conserved feature of macrophage immune responses, as it is present in both mouse and human systems. 
A
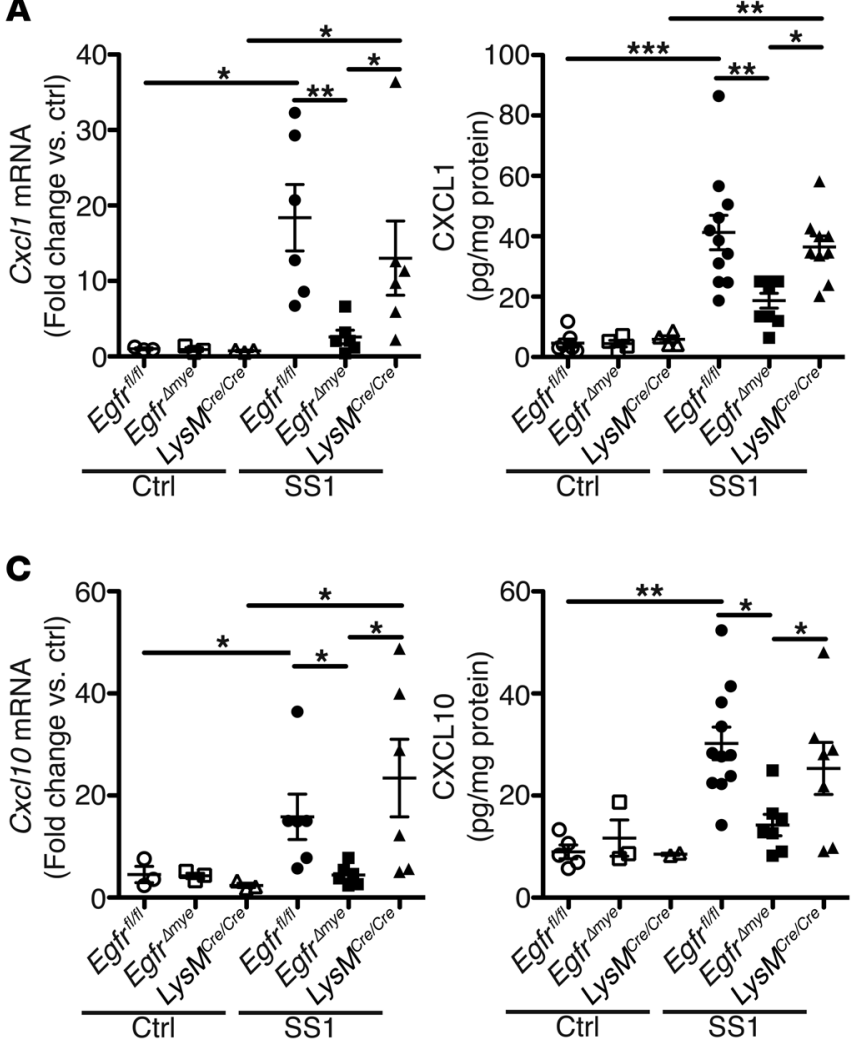

$\mathbf{E}$
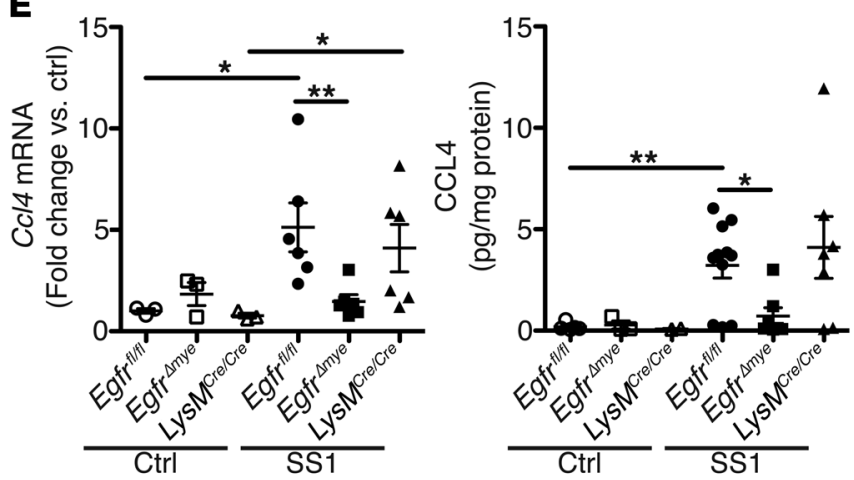
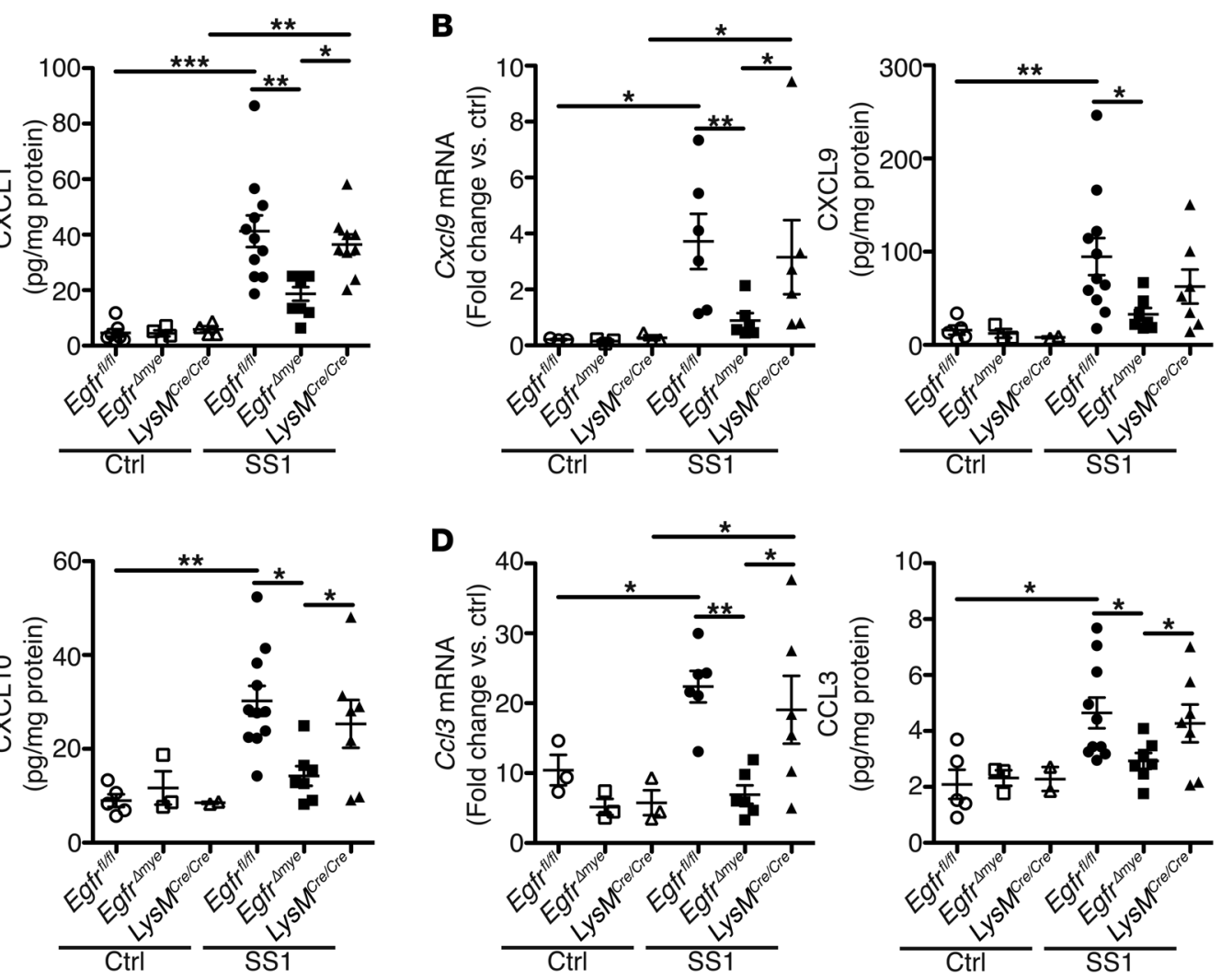

$\mathbf{F}$
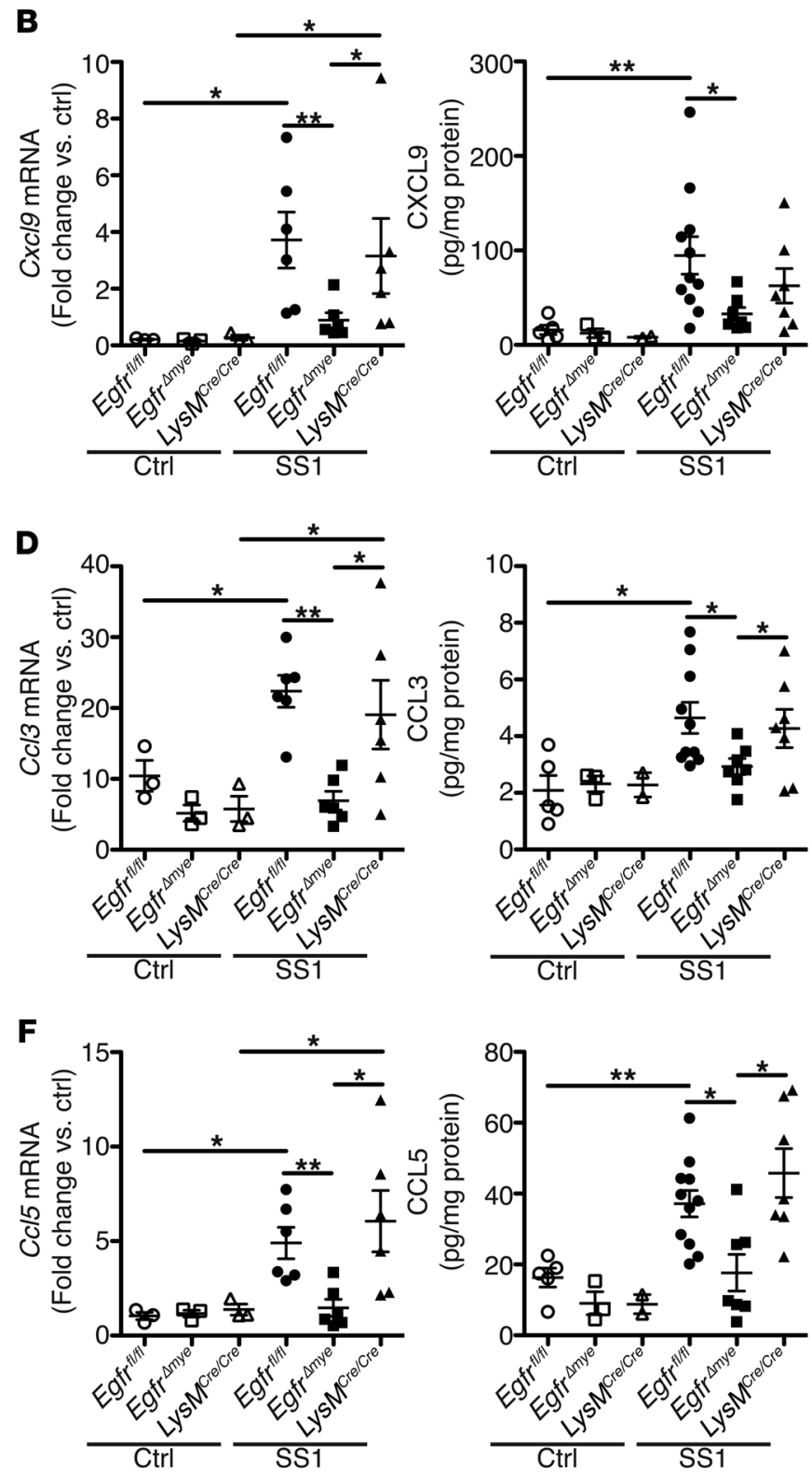

Figure 4. Egfr ${ }^{\text {myye }}$ mice have significantly decreased chemokine production in gastric tissue. mRNA and protein levels of the cytokines/chemokines (A) CXCL1, (B) CXCL10, (C) CXCL9, (D) CCL3, (E) CCL5, and (F) CCL4 were assessed by qRT-PCR and Luminex Multiplex Array, respectively, from gastric tissue 4 months p.i. with $\mathrm{H}$. pylori SS1. ${ }^{*} P<0.05$ and ${ }^{*} P<0.01$, and ${ }^{* *} P<0.001$. Statistical significance for $\mathbf{A}-\mathbf{F}$ was calculated by 1 -way ANOVA with the Kruskal-Wallis post test, followed by the Mann-Whitney $U$ test. In all panels, $n=2-5$ uninfected and 6-11 infected mice per genotype.

EGFR activation can occur in either a ligand-dependent or ligand-independent manner $(42,43)$. Previous studies in epithelial cells have revealed that p-Y1068 is a marker of ligand-dependent activation and p-S1046/47 is a marker of ligand-independent activation $(42,43)$. Since our data indicate that $H$. pylori infection leads to the phosphorylation of both residues, we investigated activation mechanisms in macrophages. Heparin-binding EGF (HB-EGF) is primarily produced by macrophages and is an EGFR ligand (44). TNF- $\alpha$ is a known inducer of ligand-independent EGFR activation $(42,45)$. Treatment of WT BMmacs with an anti-HB-EGF-neutralizing $\mathrm{Ab}$ resulted in a modest inhibition of $\mathrm{H}$. pylori-induced EGFR phosphorylation (Figure 2G). However, treatment of macro- phages with anti-TNF- $\alpha$-neutralizing Ab ablated EGFR activation at p-Y1068 and p-S1046/47 (Figure 2G and Supplemental Figure 4). Moreover, treatment with recombinant TNF- $\alpha$ also stimulated phosphorylation at S1046/47 (Supplemental Figure 4). These data suggest that EGFR can be activated in a ligand-independent, TNF- $\alpha$ dependent mechanism in macrophages during $H$. pylori infection.

Macrophage EGFR signaling is critical for controlling bacterial burden and mounting an adequate immune response to infection. To determine the role of EGFR signaling in macrophages in vivo, we obtained Egfrol/fl mice crossed with $L y s M^{\mathrm{Cre} / \mathrm{Cre}}$ mice, creating the Egfrumye mouse (19). The LysM driver leads to the excision of the Egfr alleles that are flanked by loxp sites in myeloid cells $(19,46)$. 
A

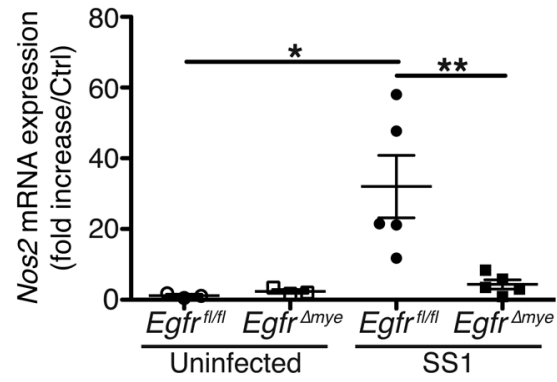

B

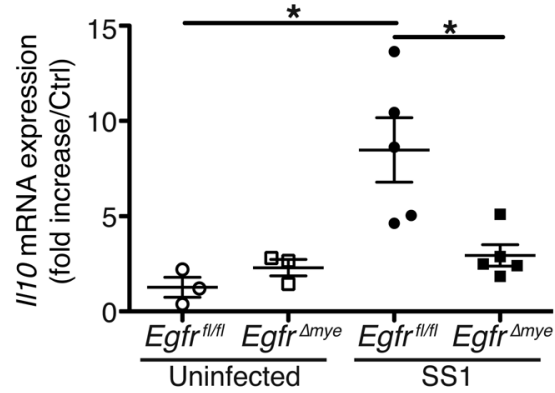

C

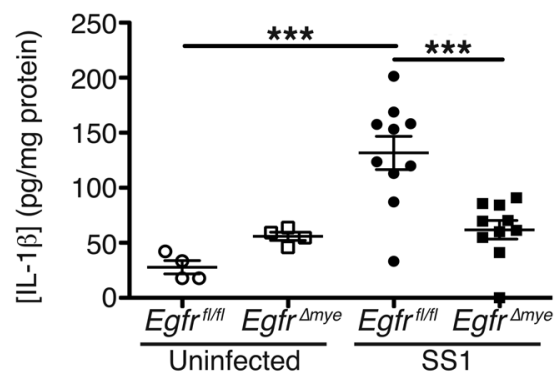

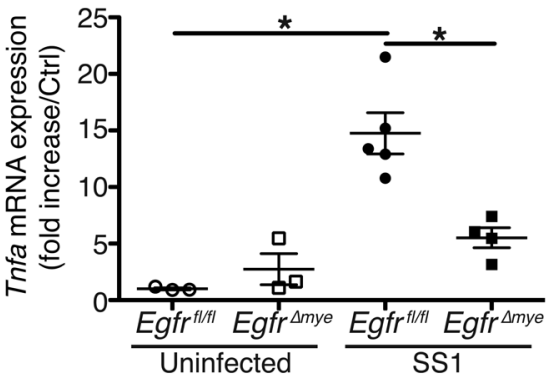
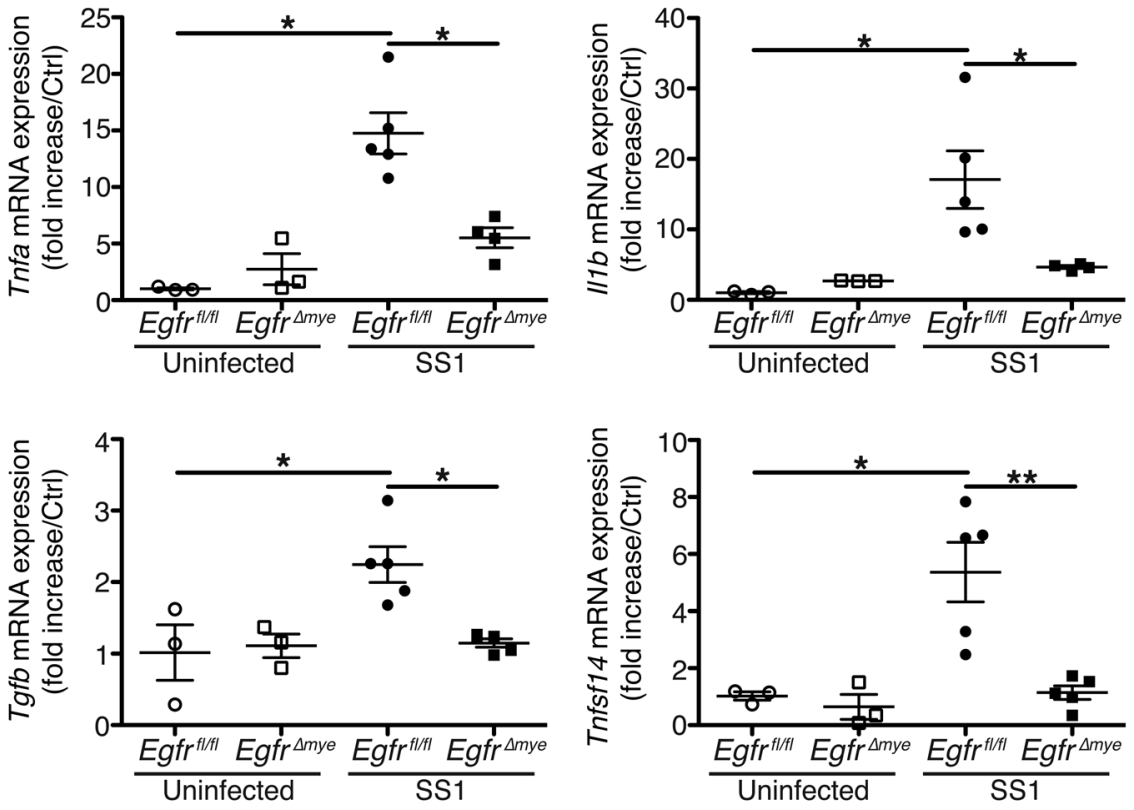

D

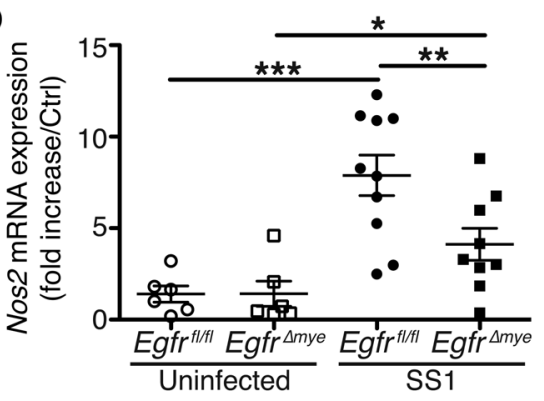

E

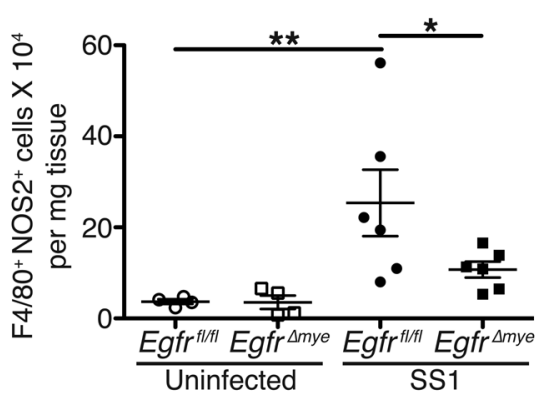

Figure 5. Egfr ${ }^{\text {mye }}$ mice have significantly decreased $M 1$ and Mreg cytokine production in gastric tissue and Gmacs. (A) mRNA levels of the proinflammatory cytokines Nos2, Tnfa, and I/1b were assessed by qRT-PCR from gastric tissue 4 months p.i. with H. pylori SS1. ${ }^{*} P<0.05$ and ${ }^{* *} P<0.01$. (B) mRNA levels of the antiinflammatory cytokines I/10, Tgfb, and Tnfsf14 (Light) were assessed by qRT-PCR from gastric tissue 4 months p.i. with $H$. pylori SS1. $n=$ 3 uninfected mice and 5 infected mice per genotype (A and $\mathbf{B}$ ). ${ }^{*} P<0.05$ and ${ }^{* *} P<0.01$. Statistical significance in $\mathbf{A}$ and $\mathbf{B}$ was calculated by 1 -way ANOVA with the Kruskal-Wallis post test, followed by the Mann-Whitney $U$ test. (C) Measurement of IL-1 $\beta$ by ELISA in gastric tissues 4 months p.i. with $H$. pylori SS1. $n=4$ uninfected and 10 infected mice per genotype. ${ }^{* *} P<0.001$. (D) F4/80+ Gmacs were magnetically selected from the lamina propria at 48 hours p.i. with $H$. pylori SS1 and mRNA levels of Nos2 were assessed by qRT-PCR. $n=6$ uninfected and $9-10$ infected mice per genotype. ${ }^{*} P<0.05,{ }^{* *} P<0.01$, and ${ }^{* *} P<0.001$. Statistical significance in $\mathbf{C}$ and $\mathbf{D}$ was calculated by 1-way ANOVA with Newman-Keuls post test. (E) Protein levels of NOS2 were assessed in $\mathrm{F} 4 / 80^{+}$Gmacs 48 hours p.i. with $\mathrm{H}$. pylori SS1. $n=4$ uninfected and 6 infected mice per genotype. ${ }^{*} P<0.05$ and ${ }^{* *} P<0.01$, by 1 -way ANOVA with the Kruskal-Wallis test, followed by the Mann-Whitney $U$ test.

We determined knockdown of EGFR in several ways. First, Egfrit/l and Egframye BMmacs were isolated and infected with $H$.pylori PMSS1 for 24 hours; DNA was isolated, and excision of the Egfr gene was detected (Supplemental Figure 5A). Second, significant knockdown of total EGFR (t-EGFR) protein levels was demonstrated in Egfrimye BMmacs compared with Egfir/l/ BMmacs, both before and after infection with $H$. pylori (Supplemental Figure 5, B and C).

Third, previous studies have demonstrated that the peak of macrophage infiltration is 48 hours p.i. with $\mathrm{H}$. pylori $(47,48)$. Using flow cytometry and F4/80 and CD11b as macrophage markers, the number of t-EGFR ${ }^{+}$Gmacs was significantly decreased in Egfromye mice versus Egfrol/f mice at this time point (Supplemental Figure 5D). Further, the number of infiltrating Gmacs was not different between genotypes (Supplemental Figure 5E). The number of pan-cytokeratin ${ }^{+}$-EGFR ${ }^{+}$gastric epithelial cells was also not different (Supplemental Figure 5F), indicating the specificity of the Lys $M$-Cre driver. The use of an isotype control $\mathrm{Ab}$ verified the specificity of t-EGFR staining (Supplemental Figure 5G).

Last, we determined the expression levels of lysozyme 2 (Lysm, also known as $L y z 2$ ), the gene on which the Cre recombinase was placed, and of Cre itself. Gastric tissues at 4 months p.i. and BMmacs infected with $H$. pylori demonstrated that, while the Egfill/l mice expressed Lysm, the Egfrume mice had no Lysm expression (Supplemental Figure 6), verifying the durability of the Lysm knockout. Further, there were very high levels of Cre mRNA both before and after chronic infection, confirming that there was no loss of Cre expression during our experiments (Supplemental Figure 6).

Having established that EGFR is effectively deleted only in macrophages before and after infection, we used our established model of chronic infection with $H$. pylori SS1 for 4 months $(32,33,49)$. Egfrumye mice showed significantly decreased histologic gastritis after a 4-month period of H. pylori SS1 infection (Figure 3, A and B) and 
A

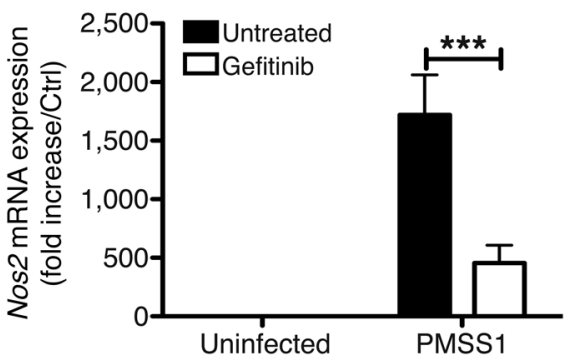

B

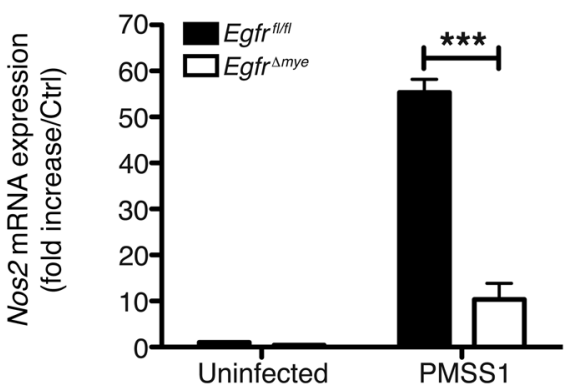

C

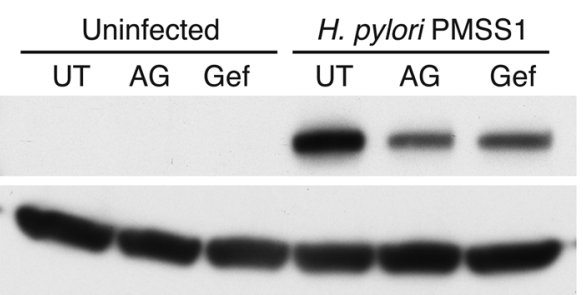

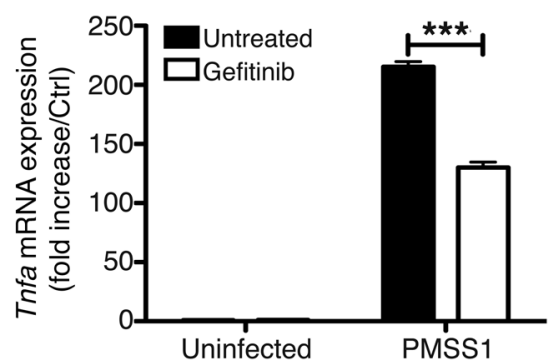
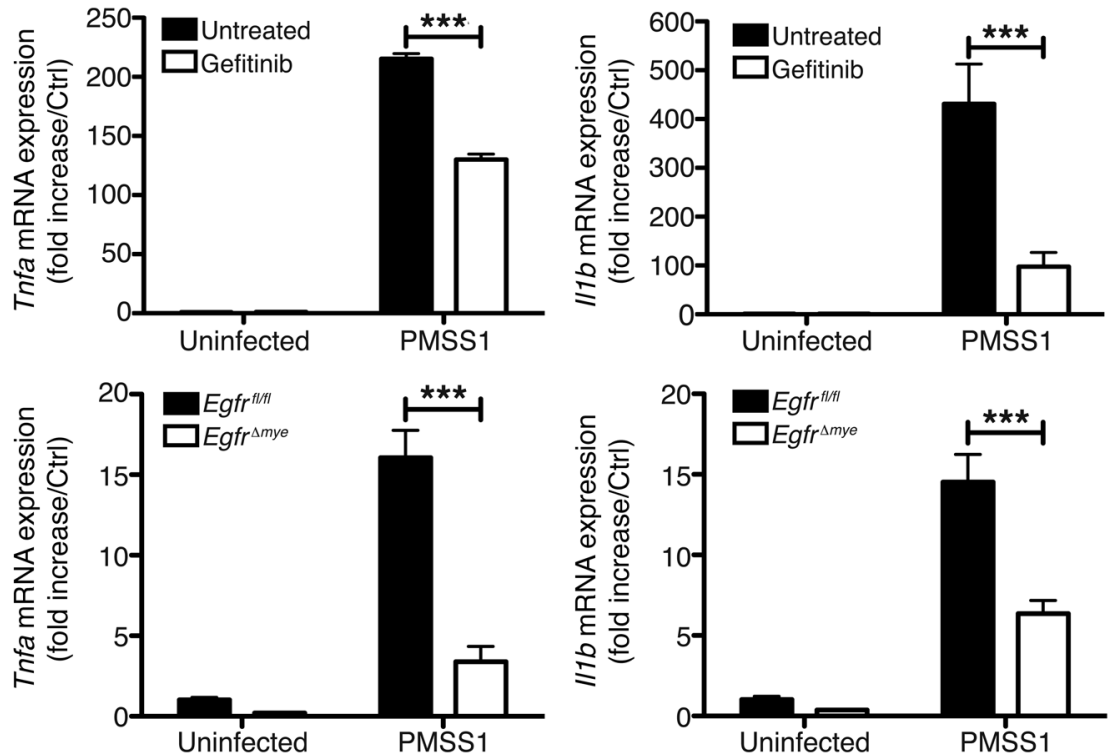

NOS2

D
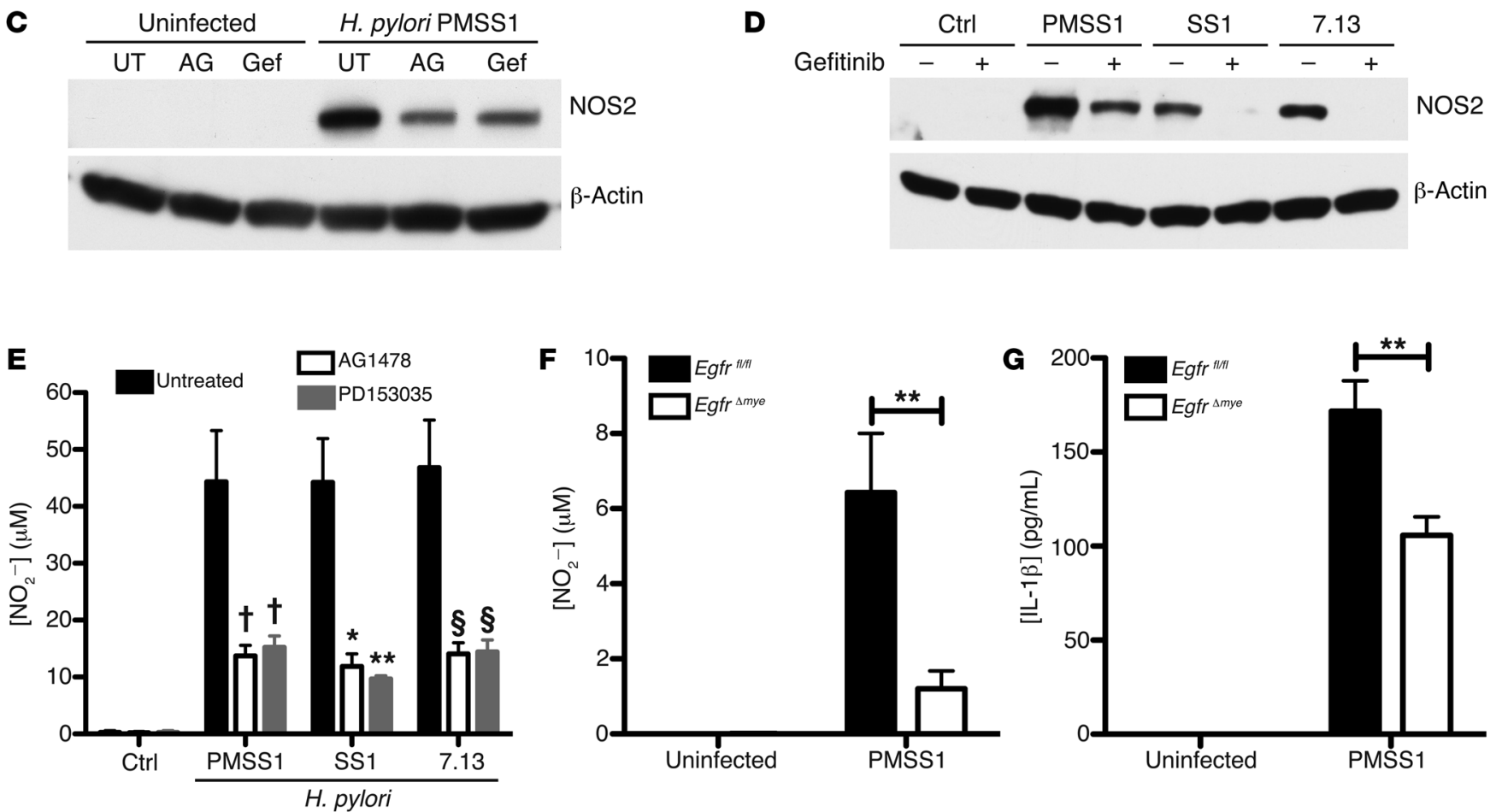

Figure 6. EGFR signaling is critical for macrophage activation and function. (A) mRNA levels of the M1 activation markers Nos2, Tnfa, and I/1b were assessed by qRT-PCR in WT BMmacs 24 hours p.i. with H. pylori PMSS1 $\pm 10 \mu \mathrm{M}$ gefitinib. $n=3$ biological replicates. ${ }^{* * *} P<0.001$. (B) mRNA levels of the M1 activation markers Nos2, Tnfa, and II1b were assessed by qRT-PCR in Egffllffl and Egfr ${ }^{\lrcorner m y e}$ BMmacs 24 hours p.i. with $H$. pylori PMSS1. $n=3$ mice per genotype. ${ }^{* *} P<0.001$. (C) Representative Western blot of NOS2 in RAW 264.7 cells 24 hours p.i. with H. pylori PMSS1 \pm 150 nM AC1478 (AG) or $10 \mu M$ gefitinib (Gef). UT, untreated. $n=3$ biological replicates. (D) Representative Western blot of NOS2 in WT BMmacs 24 hours p.i. with H. pylori PMSS1, SS1, or 7.13 \pm 10 $\mu \mathrm{M}$ gefitinib. $n=3$ biological replicates. (E) Measurement of $\mathrm{NO}_{2}^{-}$from RAW 264.7 cell supernatants 24 hours p.i. with $H$. pylori PMSS1, SS1, or $7.13 \pm 150 \mathrm{nM}$ AG1478 or $300 \mathrm{nM}$ PD153035. $n=5$ biological replicates. ${ }^{\dagger} P<0.05$ versus PMSS1 alone; ${ }^{*} P<0.05$ and ${ }^{* *} P<0.01$ versus SS1 alone; ${ }^{\S} P<0.05$ versus 7.13 alone. (F) Measurement of $\mathrm{NO}_{2}{ }^{-}$from Egfr $\mathrm{r}^{\mathrm{fl} / \mathrm{fl}}$ and $E g f \mathrm{fr}^{\mathrm{Imye}} \mathrm{BMmac}$ supernatants 24 hours p.i. with $\mathrm{H}$. pylori PMSS1. $n=3$ mice per genotype. ${ }^{* *} P<0.01$. (C) Measurement of IL-1 $\beta$ in Egfrl/fl and Egfr ${ }^{\lrcorner m y e}$ BMmac supernatants 24 hours p.i. with $H$. pylori PMSS1. $n=3$ mice per genotype. ${ }^{* *} P<0.01$. Statistical significance in all panels was calculated by 1-way ANOVA with Newman-Keuls post test.

decreased acute gastritis after a 1-month period of $H$. pylori PMSS1 infection (Supplemental Figure 7A). Previous reports have indicated that gastric inflammation, as a marker of immune response, is associated with control of $H$. pylori load in mouse models $(50,51)$. As such, there was increased $H$. pylori colonization in Egfr ${ }^{\text {mmye }}$ mice compared with that observed in Egfirl/f and $L y s M^{\mathrm{Cre} / \mathrm{Cre}}$ mice (Figure 3C). Increased colonization in Egfrimye mice was also observed in the 1-month model of infection with H.pylori PMSS1 (Supplemental Figure 7B). 
A

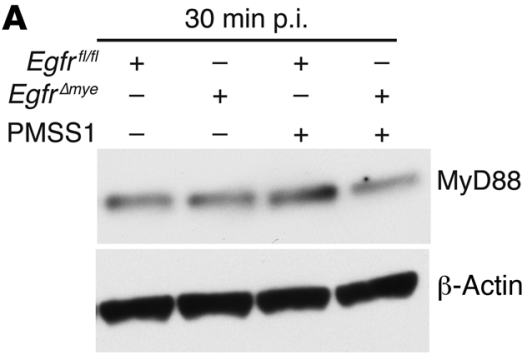

C

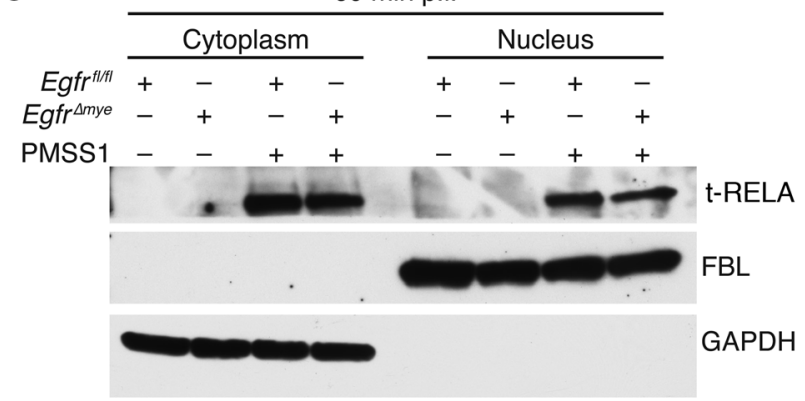

E

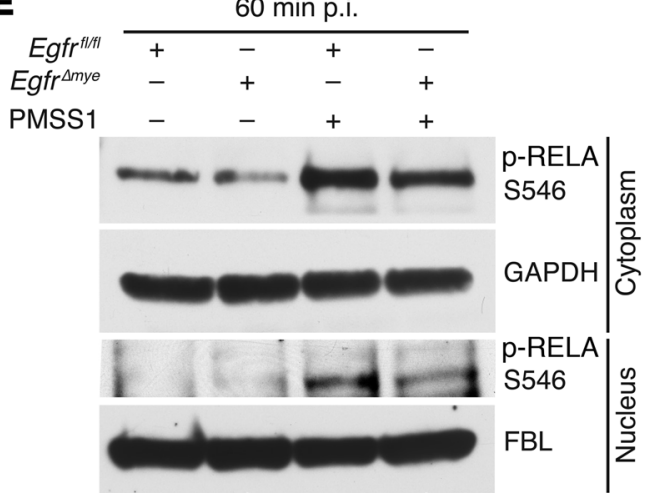

B

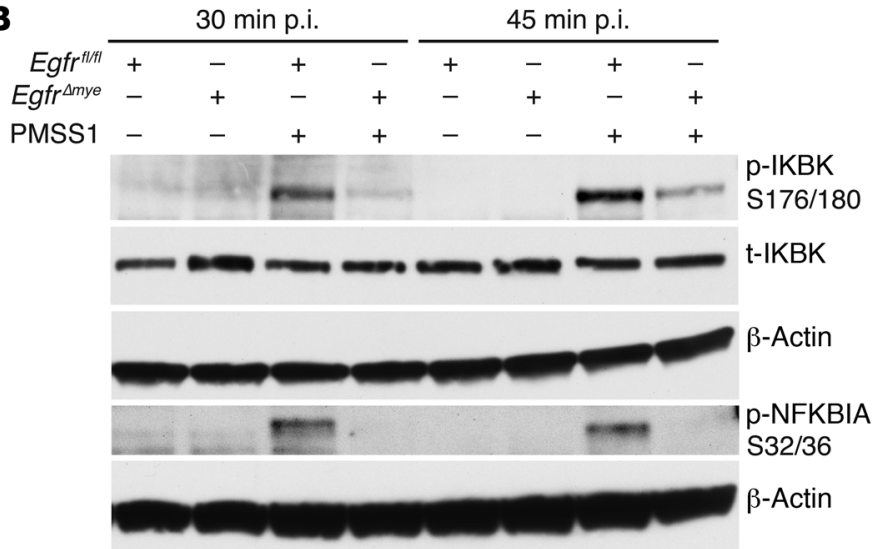

D
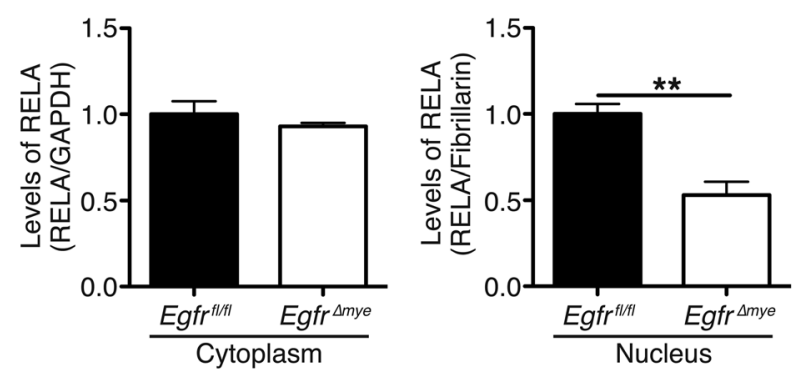

$\mathbf{F}$

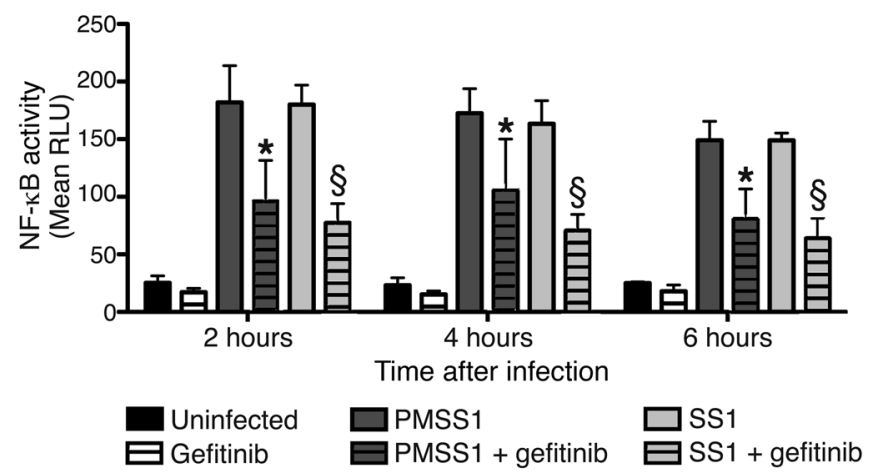

Figure 7. EGFR and NF- $\kappa B$ form a critical link in macrophages in response to H. pylori. (A) Representative Western blot of MyD88 protein levels in Egfr fl/fl and Egfr ${ }^{\text {\mye }}$ BMmacs 30 minutes p.i. with H. pylori PMSS1. $n=3$ biological replicates. (B) Representative Western blots of $p$-IKBK (also known as $p$-IKK) and p-NFKBIA (also known as p-IKB $\alpha$ ) protein levels in Egfr fl/fl and Egfr ${ }^{\Delta m y e}$ BMmacs 30 and 45 minutes p.i. with H. pylori PMSS1. $n=3$ biological replicates. (C) Representative Western blot of cytoplasmic and nuclear fractions of t-RELA (p65) in Egfr ${ }^{f / f l}$ and Egfr ${ }^{\text {Imye }}$ BMmacs 60 minutes p.i. with $H$. pylori PMSS1. $n=3$ biological replicates. (D) Densitometric analysis of cytoplasmic and nuclear t-RELA. $n=3$ biological replicates. ${ }^{*} P<0.01$, by 2 -tailed Student's $t$ test. (E) Western blot of cytoplasmic and nuclear fractions of p-RELA (p-p65) in Egfr fl/fl and Egfr ${ }^{\text {\mye }}$ BMmacs 60 minutes p.i. with H. pylori PMSS1. (F) Measurement of luminescence from an NF-KB luciferase reporter in immortalized BMmacs (NCL cells) at the indicated time points p.i. with $H$. pylori PMSS1 or SS1 $\pm 10 \mu$ M gefitinib. $n=4$ biological replicates ${ }^{*} P<0.05$ versus PMSS1 alone; ${ }^{\circledR} P<0.05$ versus $S S 1$ alone, by 1 -way ANOVA with Newman-Keuls post test.

$\mathrm{CD} 68^{+}$Gmacs from infected gastric tissues in Egfrfl/fl and

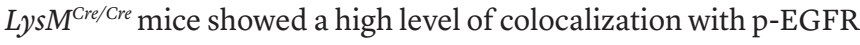
in situ, indicating that $H$. pylori infection induces EGFR signaling in macrophages (Figure 3D). $\mathrm{CD}^{+} 8^{+}$Gmacs in infected Egfrumye gastric tissues did not have any detectable p-EGFR (Figure 3D). Moreover, $\mathrm{CD}^{2} 8^{+} \mathrm{Gmacs}$ in infected Egfr ${ }^{\text {smye }}$ gastric tissues did not have any demonstrable levels of t-EGFR, while CD $68^{+} \mathrm{Gmacs}$ from $E g f r^{f / f l}$ and $L y s M^{C r e / C r e}$ mice had high levels of t-EGFR (Figure $3 \mathrm{E})$. These data indicate that the deletion of EGFR is maintained throughout chronic $H$. pylori infection.

To determine whether our findings were generalizable to another enteric bacterial infection, Egfrl/fll and Egfr ${ }^{\lrcorner m y e}$ mice were inoculated with $C$. rodentium for 14 days, which is a model of colitis (52). Egfr ${ }^{\text {umye }}$ mice showed increased C. rodentium bacterial burden in colonic tissue (Supplemental Figure 8A). When compared with Egfrl/fl mice, Egfrumye mice were protected from weight loss, indicative of decreased clinical disease severity (Supplemental Figure 8B). Decreased disease severity, combined with increased colonization, in the $C$. rodentium model is concordant with the findings in the $H$. pylori model above. $\mathrm{CD}^{+} 8^{+}$macrophages from infected colonic tissues in Egfrf/fl mice demonstrated a high level of colocalization with p-EGFR, indicating that $C$. rodentium infection also induces EGFR signaling in macrophages (Supplemental Figure $8 \mathrm{C}$ ). $\mathrm{CD}^{+} 8^{+} \mathrm{p}-\mathrm{EGFR}^{+}$colonic macrophages were not pres- 
A

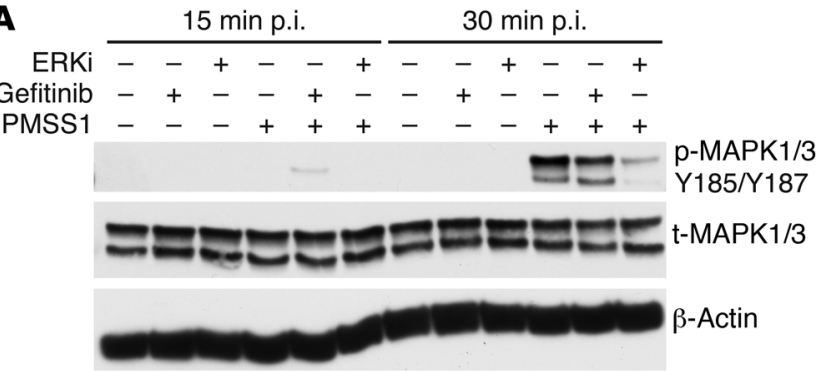

C

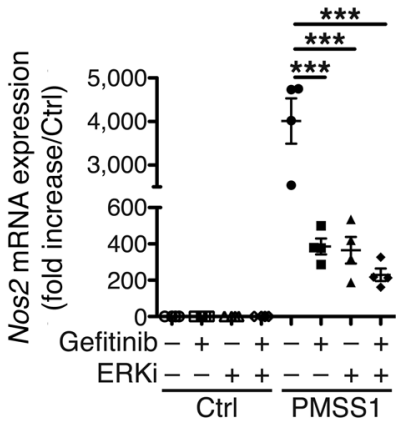

E

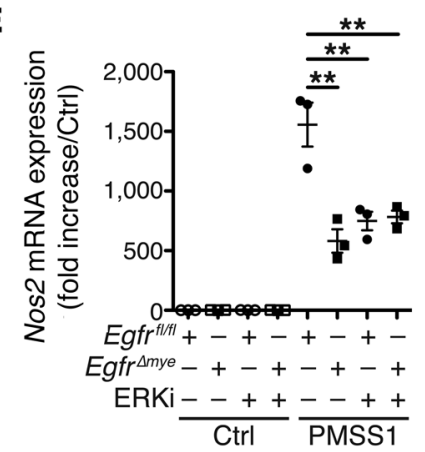

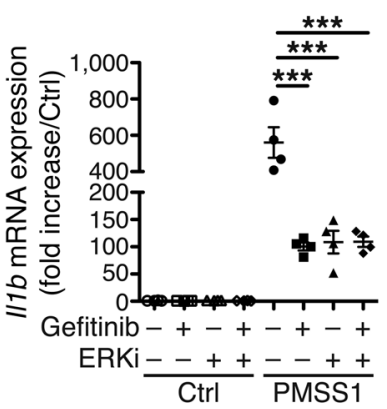

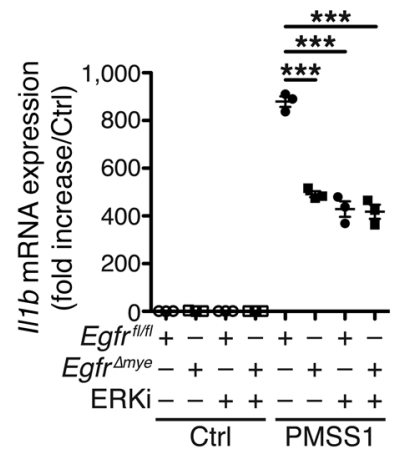

B

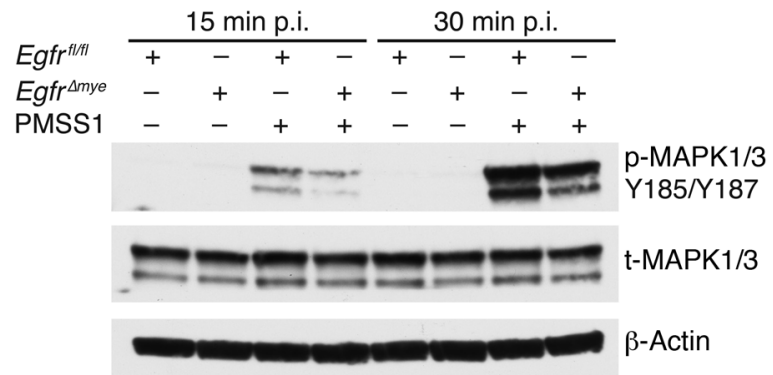

D
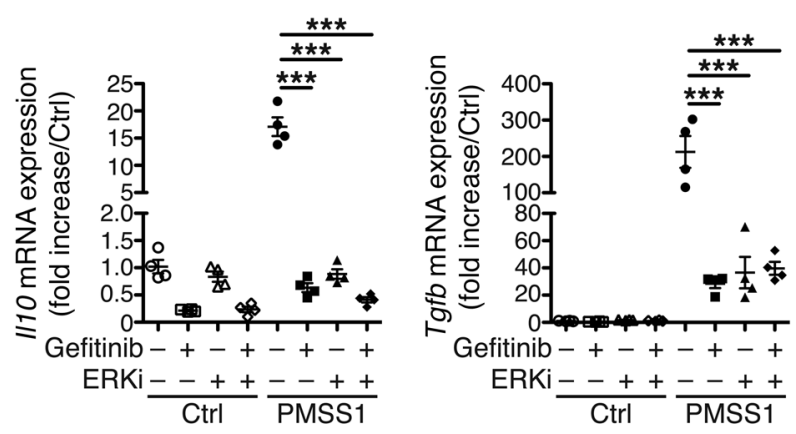

F
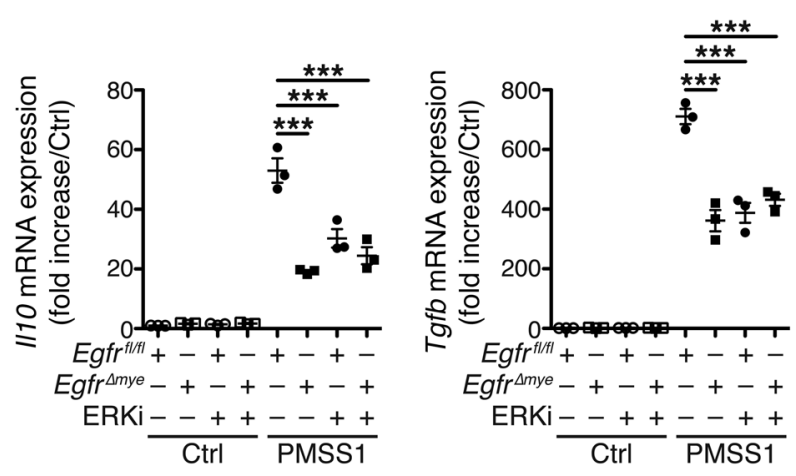

Figure 8. EGFR signaling and MAPK1/3 signaling are linked in regulating macrophage activation in response to $\boldsymbol{H}$. pylori. (A) Representative Western blot of p-MAPK1/3 levels in WT BMmacs $\pm 10 \mu \mathrm{M}$ gefitinib or $\pm 50 \mu \mathrm{M}$ ERKi at 15 and 30 minutes p.i. with $\mathrm{H}$. pylori PMSS1. $n=3$ biological replicates. (B) Representative Western blot of p-MAPK1/3 levels in Egfr ${ }^{f l / f l}$ and Egfr ${ }^{\Delta \text { mye }}$ BMmacs $\pm 50 \mu$ M ERKi at 15 and 30 minutes p.i. with $\mathrm{H}$. pylori PMSS1. $n=3$ biological replicates. (C) mRNA levels of the M1 activation markers Nos2 and $/ 11 \mathrm{~b}$ were assessed by qRT-PCR in WT BMmacs $\pm 10 \mu \mathrm{M}$ gefitinib and/or $\pm 50 \mu \mathrm{M}$ ERKi 24 p.i. with H. pylori PMSS1. $n=5$ mice. ${ }^{* *} P<0.001$. (D) mRNA levels of the Mreg activation markers $\| 10$ and Tgfb were assessed by qRT-PCR in WT BMmacs $\pm 10 \mu \mathrm{M}$ gefitinib and/or $\pm 50 \mu \mathrm{M}$ ERKi 24 p.i. with $\mathrm{H}$. pylori PMSS1. $n=5$ mice. ${ }^{* *} P<0.001$. (E) mRNA levels of the M1 activation markers Nos2 and $/ 11 \mathrm{~b}$ were assessed by qRT-PCR in Egfr ${ }^{f l / f l}$ and Egfr ${ }^{\text {\mye }}$ BMmacs $\pm 50 \mu$ M ERKi 24 p.i. with $H$. pylori PMSS1. $n=3$ mice per genotype. ${ }^{* *} P<0.01$ and ${ }^{* * *} P<0.001$. (F) mRNA levels of the Mreg activation markers $/ 110$ and Tgfb were assessed by qRT-PCR in Egfrl/fl and Egfr ${ }^{\text {Imye }}$ BMmacs $\pm 50 \mu M$ ERKi 24 p.i. with $H$. pylori PMSS1. $n=3$ mice per genotype. ${ }^{* *} P<0.001$. Statistical significance in $\mathbf{C}-\mathbf{F}$ was calculated by 1-way ANOVA with Newman-Keuls post test.

ent in infected Egframye colonic tissues, again confirming effective Egfr excision in these mice (Supplemental Figure 8C). These data further indicate that EGFR signaling in macrophages is important in the host response to enteric infections.

EGFR signaling is critical for proinflammatory chemokine production in vivo. Given our in vivo findings, we hypothesized that the loss of Egfr in myeloid cells diminished the innate immune responses to bacterial pathogens. To assess host immune responses, we used a Luminex Multiplex Array to assess 32 chemokines and cytokines. Six analytes were significantly decreased in gastric tissues from H. pylori-infected Egfrumye mice compared with gastric tissues from Egfirl/fl or $L y s M^{\text {Cre/Cre }}$ mice (Figure 4). Moreover, all of these analytes, C-X-C motif ligand 1 (CXCL1, also known as GRO $\alpha$ or KC); CXCL9 (also known as MIG); CXCL10 (also known as IP-10); C-C motif ligand 3 (CCL3, also known as MIP-1 $\alpha$ ); CCL4 (also known as MIP-1 1 ); and CCL5 (also known as RANTES), were altered at both the MRNA and protein levels (Figure 4), indicating that loss of EGFR in myeloid cells leads to a significant ablation of the innate immune response at the transcriptional level. Alterations in chemokine responses further indicate that macrophage EGFR signaling is necessary for the gastric inflammatory response to $H$. pylori. Cytokines that were either not significantly different between genotypes, not increased by $H$. pylori infection, or not detected are listed in Supplemental Table 1.

EGFR signaling is critical for proinflammatory cytokine production and Nos 2 expression by macrophages in vivo. Given the high degree of colocalization of p-EGFR and $\mathrm{CD} 68^{+}$macrophages in both gastric and colonic tissues during infection and the fact that macrophages are known to be major drivers of $H$. pylori-induced inflammation (34), we next examined genes typically expressed 
A
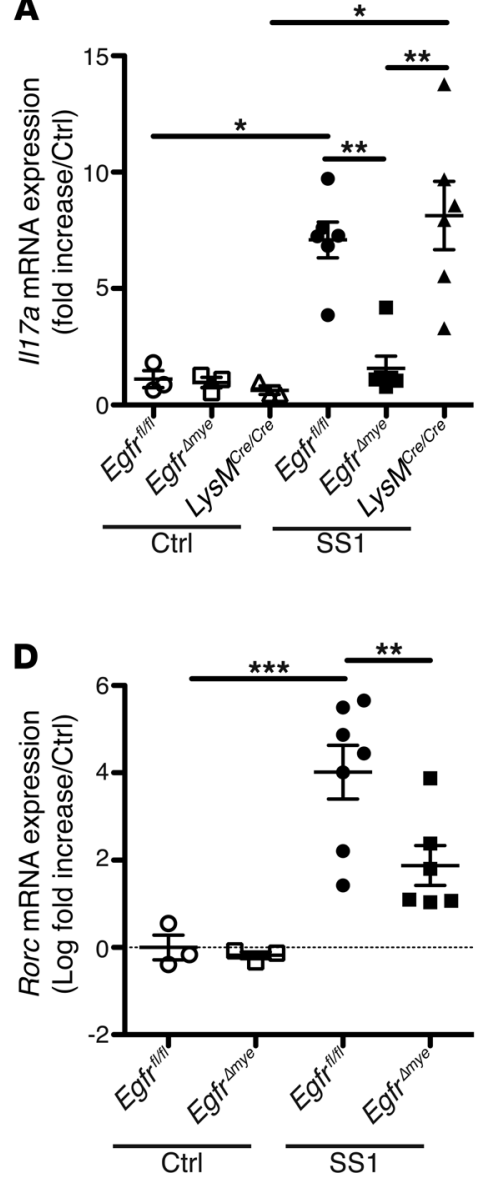

B
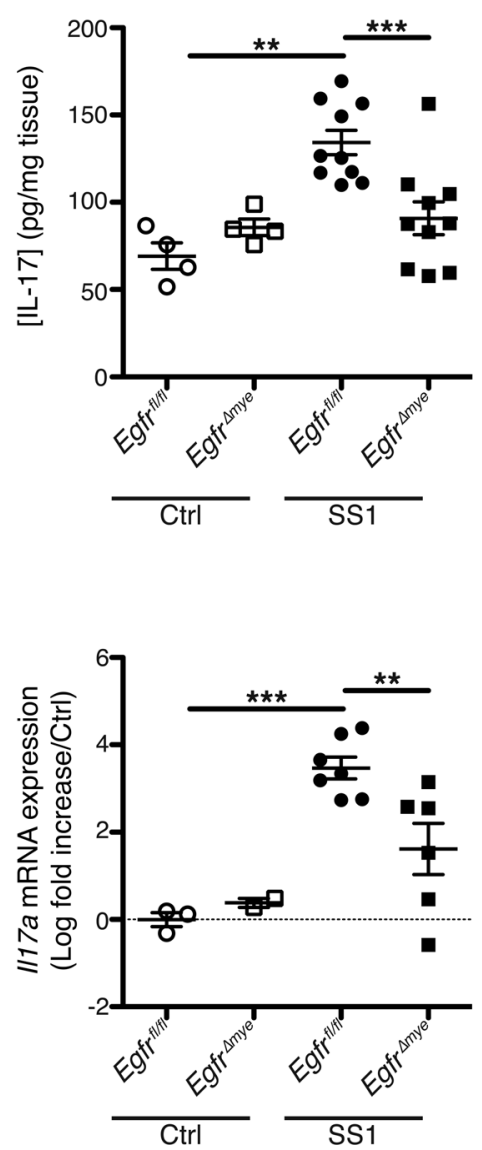

C

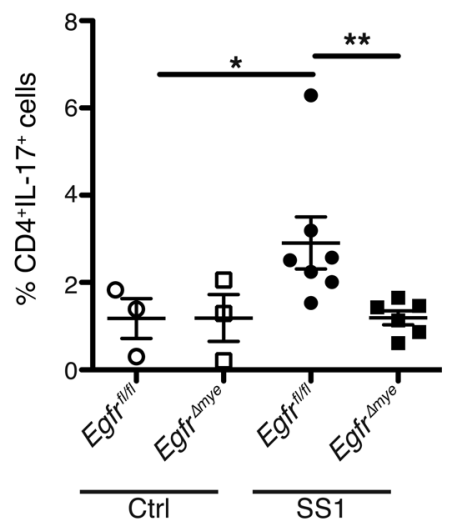

Figure 9. EGFR deficiency in macrophages leads to a diminished Th17 response to $\boldsymbol{H}$. pylori. (A) mRNA levels of $I 117 a$ were assessed by qRT-PCR in gastric tissues from Egfr ${ }^{f l / f f}$, Egfr ${ }^{\Delta m y e}$, and LysM ${ }^{\text {cre/ } / \text { re }}$ mice 4 months p.i. with H. pylori SS1. $n=3$ uninfected and 6 infected mice per genotype. ${ }^{*} P<0.05$ and ${ }^{* *} P<0.01$. (B) IL-17 protein levels were assessed by ELISA in gastric tissues from Egfr fl/fl and Egfr ${ }^{\mathrm{fmye}}$ mice 4 months p.i. with $\mathrm{H}$. pylori SS1. $n=4$ uninfected and 10 infected

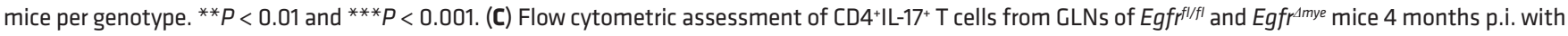
H. pylori SS1. Isolated T cells were cultured in 96-well plates containing $5 \mu \mathrm{g} / \mathrm{ml}$ anti-CD3 and $1 \mu \mathrm{g} / \mathrm{ml}$ anti-CD28. Cells were then stimulated with $20 \mathrm{ng} / \mathrm{ml}$ PMA and $1 \mu \mathrm{g} / \mathrm{ml}$ ionomycin for 4 hours. $n=3$ uninfected and 7 infected mice per genotype. ${ }^{*} P<0.05$ and ${ }^{* *} P<0.01$. (D) mRNA levels of the Th17 markers Rorc and II17 were assessed by qRT-PCR from magnetically selected CD4+ T cells from the gastric lamina propria of Egfr/f/fl and Egfr ${ }^{\mathrm{Imye}}$ mice 4 months p.i. with H. pylori SS1. $n=3$ uninfected and 6-7 infected mice per genotype. ${ }^{* *} P<0.01$ and ${ }^{* *} P<0.001$. Statistical significance in all panels was calculated by 1 -way ANOVA with Newman-Keuls post test.

by macrophages. Markers of M1 macrophage activation Nos2, Tnfa, and $I l 1 b$ - were all decreased in gastric tissues from H. pylori-infected Egfrumye mice compared with markers detected in Egfril/l mice (Figure 5A). Markers of Mreg activation - Il10, Tgfb, and TNF (ligand) superfamily member 14 (Tnfsf14, also known as Light) - were also decreased in infected Egfrumye gastric tissues (Figure 5B). Markers of M2 activation - arginase 1 (Arg1) and chitinase, acidic (Chia1) - were not significantly altered in Egframye mice (Supplemental Figure 9). IL-1 $\beta$ protein was also significantly decreased in H. pylori-infected Egfrumye gastric tissues (Figure 5C), supporting the mRNA data indicating a role for myeloid EGFR in regulating the M1 response to H. pylori. Furthermore, Gmacs from infected Egfrimye mice expressed lower levels of Nos2 mRNA (Figure 5D) and NOS2 protein (Figure 5E) compared with levels in Gmacs from infected Egfir/l/ mice. As there was no difference in macrophage infiltration (Supplemental Figure 4E), these data indicate that the changes in NOS2 levels are specifically due to loss of EGFR rather than alterations in macrophage numbers.
In the C. rodentium model, only M1 markers were significantly induced by infection in colon tissues from Egfril/l mice; as in H. pylori infection, expression levels of Nos 2 and Tnfa were significantly decreased in the Egfrimye mice, and Il1b showed a similar trend (Supplemental Figure 10). Thus, macrophage EGFR signaling is an important mediator of proinflammatory and antiinflammatory gene expression during gastrointestinal mucosal infection.

EGFR signaling is a key component in macrophage activation and function. Because limited numbers of Gmacs can be obtained for experimentation, we used BMmacs for further studies addressing the role of EGFR in macrophages. We also used bone marrow-derived DCs (BMDCs) to assess the role of EGFR signaling in the DC population. Differentiation of macrophages and DCs is demonstrated in Supplemental Figure 11. WT BMmacs treated with gefitinib demonstrated significantly decreased M1 activation in response to $H$. pylori, as determined by decreased expression of Nos2, Tnfa, and Il1b (Figure 6A). BMmacs from Egfrumye mice also exhibited decreased expression of these M1 genes when 
A
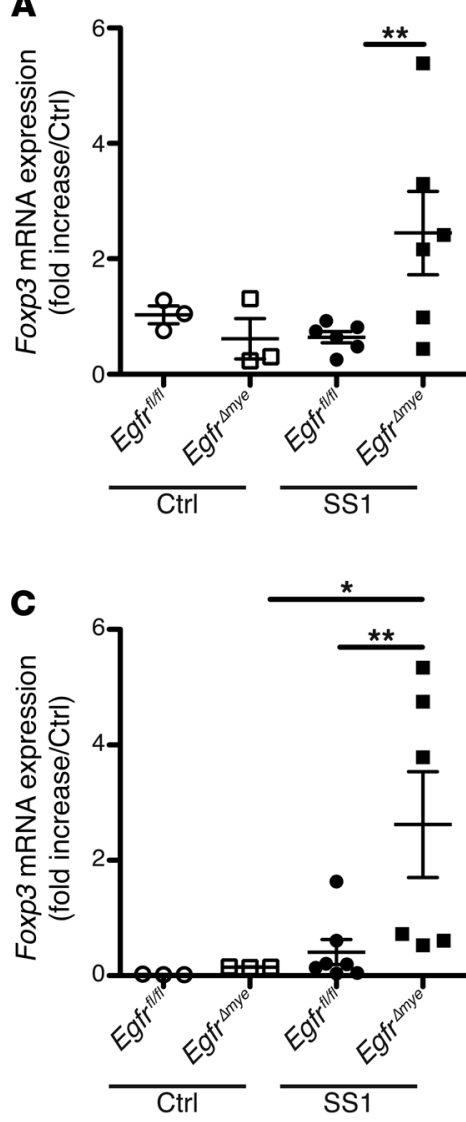

B
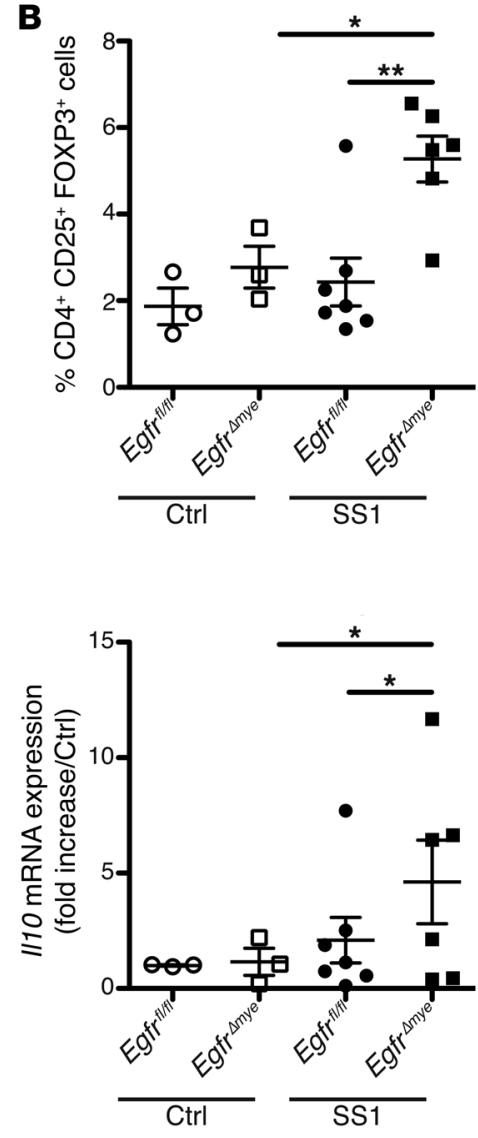

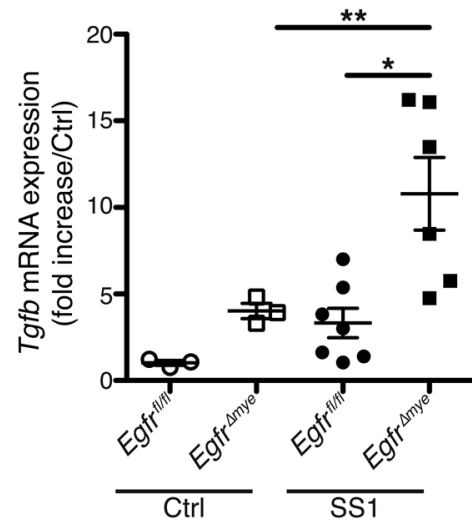

Figure 10. EGFR deficiency in macrophages leads to an enhanced Treg response during $\boldsymbol{H}$. pylori infection. (A) mRNA levels of Foxp3 were assessed by qRT-PCR in gastric tissues from Egfr fl/fl and Egframye mice 4 months p.i. with $H$. pylori SS1. ${ }^{* *} P<0.01 . n=3$ uninfected and 6 infected mice per genotype. (B) Assessment of CD4+CD25+FOXP3 ${ }^{+} T$ cells from GLNs of Egfr fl/fl and Egfr ${ }^{\text {Imye }}$ mice 4 months p.i. with $\mathrm{H}$. pylori SS1 by flow cytometry. Isolated T cells were not cultured or were stimulated as in Figure 9C. $n=3$ uninfected and 6-7 infected mice per genotype. ${ }^{*} P<0.05$ and ${ }^{* *} P<0.01$. (C) mRNA levels of the Treg markers Foxp3, II10, and Tgfb were assessed by qRT-PCR from magnetically selected CD4+ $T$ cells from the gastric lamina propria of Egfrl/fl and Egfr $r^{\Delta m y e}$ mice 4 months p.i. with $H$. pylori SS1. $n=3$ uninfected and 6-7 infected mice per genotype. ${ }^{*} P<0.05$ and ${ }^{*} P<0.01$. Statistical significance in all panels was calculated by 1-way ANOVA with Newman-Keuls post test.

compared with Egfrl/fl BMmacs (Figure 6B). As in gastric tissues, Mreg activation with $H$. pylori infection, denoted by induction of IllO and Tgfb, was decreased in both WT BMmacs treated with gefitinib and in Egframye BMmacs (Supplemental Figure 12A and 12B). M2 gene expression was not induced by $H$. pylori infection in BMmacs (Supplemental Figure 12,C and D). Similarly, M1 and Mreg markers induced by $C$. rodentium infection were decreased by inhibition of EGFR signaling (Supplemental Figure 13, A and B). These BMmac data corroborate our in vivo findings and indicate that EGFR is a key regulator of macrophage activation during bacterial infection.

To determine whether EGFR has a global role in macrophage activation, we treated Egfrl/fl and Egfr ${ }^{\Delta m y e}$ BMmacs with the prototypical stimuli for M1, M2, and Mreg activation and assessed activation by gene expression. Stimulation of BMmacs with IFN- $\gamma$ and LPS induces an M1 response (2). As with H.pylori or C. rodentium infection, an M1 stimulus induced significantly decreased expres-

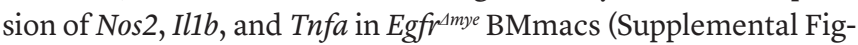
ure 14A). Neither $H$. pylori nor C. rodentium induce a significant M2 response (Supplemental Figure 9 and Supplemental Figure 12, C and D). However, Egfr ${ }^{\text {smye }}$ BMmacs activated with IL-4, a classical M2 stimulus (2), demonstrated significantly diminished mRNA expression of the M2 genes Arg1, Chia1, and resistin-like $\alpha$ (Retnla, also known as RELMa) when compared with Egfrfl/fl BMmacs (Supplemental Figure 14B). Moreover, Mreg activation, as evidenced by $I l 10$ and Tgfb expression, was significantly diminished in Egfr ${ }^{\text {smye }}$ BMmacs (Supplemental Figure 14C). Thus, EGFR has the capacity to regulate all subsets of macrophage activation under various conditions. These data further demonstrate the central role that EGFR plays in regulating macrophage activation.

H. pylori-stimulated NOS2 protein levels were also diminished in RAW 264.7 cells treated with gefitinib or AG1478, a second inhibitor of the EGFR tyrosine kinase domain (Figure 6C), or in WT BMmacs treated with gefitinib (Figure 6D). Both RAW 264.7 cells treated with EGFR inhibitors (Figure 6E) or Egfr ${ }^{\text {Amye }}$ BMmacs (Figure 6F) produced decreased NO, an effector we have shown to have antimicrobial effects $(32,53,54)$, in response to $H$. pylori. Thus, when RAW 264.7 cells were treated with gefitinib, we observed significantly increased $H$. pylori survival when bacteria were separated from macrophages by a Transwell filter support, a model that prevents phagocytosis and allows for direct measurement of the ability of macrophages to kill H. pylori 
(Supplemental Figure 15). These data are consistent with the increased bacterial survival in both the H.pylori and C. rodentium mouse models of infection with myeloid-specific deletion of Egfr. Moreover, $H$. pylori-stimulated IL-1 $\beta$ protein secretion was attenuated in Egfr ${ }^{\text {umye }}$ versus Egfrl/fl BMmacs (Figure 6G). There was no $H$. pylori strain specificity for inducing EGFR-mediated macrophage immune responses, as infection with multiple $H$. pylori strains resulted in similar findings.

Macrophages are also professional phagocytes. Therefore, we assessed the effect of EGFR signaling on macrophage phagocytosis of $H$. pylori, using a gentamicin protection assay (55) and siRNA to ensure effective loss of EGFR (Supplemental Figure 16A). We observed no difference in phagocytosis between Egfrknockdown cells and cells transfected with a nontargeting siRNA pool (Supplemental Figure 16B). Additionally, there was no difference in phagocytosis between untreated and gefitinib-treated cells, confirming our findings with Egfr siRNA (Supplemental Figure 16C). We verified that $200 \mu \mathrm{g} / \mathrm{ml}$ gentamicin effectively killed extracellular $H$.pylori, while $10 \mu \mathrm{M}$ gefitinib and $0.1 \%$ saponin had no effect on $H$. pylori viability (Supplemental Figure 16C). These findings confirmed the efficacy of this assay. Thus, EGFR signaling does not appear to play a role in bacterial phagocytosis.

EGFR is associated with resistance to apoptosis in epithelial cells (39), raising the possibility that the phenotypic effects observed in macrophages lacking EGFR are due to diminished cell survival. However, there was no difference in $H$. pylori-induced apoptosis in RAW 264.7 cells with Egfr knockdown (Supplemental Figure 17, A and B) or in those treated with gefitinib (Supplemental Figure 17C). Additionally, neither WT BMmacs, with our without gefitinib, nor Egfr ${ }^{\lrcorner m y e}$ versus Egfrfl/fl BMmacs had any differences in H.pylori-induced apoptosis (Supplemental Figure 17, D and E). Similarly, we detected no differences in annexin-V/7AAD levels in $\mathrm{F} 4 / 80^{+} \mathrm{CD}_{11} \mathrm{~b}^{+}$Gmacs from H. pylori-infected Egfrflfl or Egfr ${ }^{\text {smye }}$ mice (Supplemental Figure 17F). Importantly, there were no differences in the number of Gmacs between genotypes, further indicating that the cells are viable with or without EGFR (Supplemental Figure 17G). Moreover, we detected no differences in cleaved caspase 3 levels in $\mathrm{CD} 68^{+} \mathrm{Gmacs}$ in tissues from Egfrl/fl and Egfrumye mice 4 months p.i., as assessed by immunofluorescence (Supplemental Figure 17H). Taken together, these data indicate that EGFR does not regulate cell viability in macrophages and further confirm that the effects of Egfr deletion on macrophage phenotype are due solely to the loss of EGFR.

As our mouse model is a myeloid-specific knockout of EGFR, we also examined the expression of DC cytokines under conditions in which EGFR signaling was inhibited. WT BMDCs treated with gefitinib showed no differences in H. pylori-induced mRNA expression of the relevant cytokines Tnfa, Il6, Il12b, and Il1O (Supplemental Figure 18A). Similarly, Egfr ${ }^{\Delta m y e}$ BMDCs demonstrated no difference in H. pylori-stimulated expression of these same cytokines when compared with expression levels in Egfrfl/fl BMDCs (Supplemental Figure 18B). Moreover, Egfr ${ }^{4 m y e}$ BMDCs expressed the same level of surface MHC II during H. pylori infection as that detected in Egfroflfl BMDCs (Supplemental Figure 18, C and D). Taken together, these data indicate that EGFR signaling does not regulate DC cytokine production or antigen presentation.
EGFR signaling and NF- $\kappa B$ signaling form an important link in macrophages that is necessary for cytokine production. To further address the downstream effects of EGFR in macrophages, we examined the NF- $\mathrm{B}$ signaling pathway, a key mediator of cytokine production and innate immune responses to pathogens. $H$. pylori infection leads to NF- $\mathrm{KB}$ signaling, directly resulting in the production of cytokines and chemokines $(56,57)$. Because we are studying bacterial pathogens, we examined the classical $\mathrm{NF}-\kappa \mathrm{B}$ signaling pathway that includes myeloid differentiation primary response 88 (MyD88), inhibitor of $\kappa$ light polypeptide gene enhancer in B cells, kinase (IKBK, also known as IKK), and inhibitor of $\kappa$ light polypeptide gene enhancer in $\mathrm{B}$ cells, inhibitor $\alpha$ (NFKBIA, also known as I $\mathrm{B} \alpha$ ).

Given our findings that $H$. pylori-induced phosphorylation of EGFR was present at 15 minutes p.i. and abundant at 30 minutes p.i., downstream NF- $\mathrm{kB}$-related signaling was assessed beginning at the latter time point. H. pylori-infected Egfrsmye BMmacs demonstrated decreased MyD88 protein levels 30 minutes p.i. as compared with Egfifl/fl BMmacs (Figure 7A). A similar decrease was observed in WT BMmacs treated with gefitinib (Supplemental Figure 19A). Moreover, Egfrumye BMmacs had markedly decreased p-IKBK and p-NFKBIA levels at 30 and 45 minutes p.i. compared with levels detected in Egfifl/l BMmacs (Figure 7B). H. pyloriinfected WT BMmacs treated with gefitinib also had decreased p-IKBK and p-NFKBIA levels as compared with levels in untreated BMmacs (Supplemental Figure 19, B and C).

Consistent with the MyD88, p-IKBK, and p-NFKBIA data, there was significantly less $\mathrm{v}$-rel avian reticuloendotheliosis viral oncogene homolog A (RELA, also known as p65) translocation to the nucleus 60 minutes after H. pylori infection in Egfrumye BMmacs (Figure 7C). Densitometric analysis confirmed the significant decrease in nuclear RELA levels, with no change in the cytoplasmic levels of RELA (Figure 7D). A similar reduction in nuclear translocation of RELA was observed in WT BMmacs treated with gefitinib (Supplemental Figure 19, D-F). Nuclear levels of p-RELA were also decreased in Egfr ${ }^{\text {Amye }}$ BMmacs 60 minutes p.i. as compared with levels in Egfrlf/fl BMmacs (Figure 7E). Moreover, BMmacs expressing an NF- $\kappa \mathrm{B}$ luciferase reporter (NGL cells) (58, 59) demonstrated decreased $H$. pylori-stimulated NF- $\kappa B$ activity when treated with gefitinib (Figure 7F). Taken together, these data indicate that $\mathrm{p}$-EGFR is a key enhancer of macrophage NF- $\mathrm{BB}$ signaling in response to $H$. pylori infection, potentiating the production of inflammatory mediators, including cytokines and NO.

ERK signaling is also linked to EGFR signaling and contributes to macrophage activation. EGFR signaling is known to interact with many signaling pathways, including MAPK signaling $(60,61)$. Components of the MAPK signaling pathway include MAPK1 and MAPK3 (hereafter referred to as MAPK1/3; also known as ERK1/2) $(60,61)$. We have previously demonstrated that MAPK1/3 signaling has a role in regulating macrophage function in response to $H$. pylori and that H. pylori infection induces phosphorylation of MAPK1/3 (48).

EGFR inhibition (Figure $8 \mathrm{~A}$ ) or deletion (Figure $8 \mathrm{~B}$ ) resulted in decreased p-MAPK1/3 levels in BMmacs stimulated with H. pylori (Figure 8, A and B). These data suggest that EGFR activation is upstream of MAPK1/3 and that MAPK1/3 signaling is closely linked to EGFR signaling. To determine the role of MAPK1/3 signaling in macrophage activation, we used the 
MAPK1/3 inhibitor ERKi to prevent MAPK1/3 phosphorylation (Figure 8A). WT BMmacs were treated with gefitinib, ERKi, or both inhibitors in combination prior to infection with $H$. pylori. Gefitinib and ERKi each led to markedly decreased expression of Nos 2 and $I l 1 b$, markers of M1 activation (Figure 8C), and decreased expression of $I l 1 O$ and Tgfb, markers of Mreg activation (Figure 8D). Notably, the combination of gefitinib and ERKi did not further decrease M1 or Mreg gene expression (Figure 8, C and D). Together, these data suggest that EGFR and MAPK1/3 are in the same pathway and that EGFR is upstream of MAPK1/3.

Consistent with the data obtained using gefitinib and ERKi, H. pylori-infected Egfrlffl BMmacs treated with ERKi demonstrated a level of reduction of Nos2, Il1b, Il1O, and Tgfb mRNA expression that was similar to that observed in Egfrumye BMmacs not treated with ERKi (Figure 8. E and F). As with the combination of gefitinib and ERKi, Egfr ${ }^{4 m y e}$ BMmacs treated with ERKi did not have any further decrease in M1 or Mreg gene expression (Figure 8, E and F). These data further support the concept that EGFR and MAPK $1 / 3$ are in the same pathway(s) and exert potent effects on macrophage activation in response to $H$.pylori infection.

Macrophage EGFR is necessary for regulating both Th17 and Treg $T$ cell responses during $H$. pylori infection. As macrophages are antigen-presenting cells and play a role in initiating the adaptive immune response during $H$. pylori infection, we sought to determine the nature of the interaction between Egfr ${ }^{\Delta m y e}$ macrophages and $\mathrm{T}$ cells. Because we detected attenuated chronic inflammation at 4 months p.i. in Egfr ${ }^{\text {mye }}$ mice, we focused on this time point for detailed analysis of the adaptive immune response. We used 2 sources of $\mathrm{T}$ cells for these studies: magnetically selected $\mathrm{CD} 4^{+} \mathrm{T}$ cells from the lamina propria of the mouse stomach that were then analyzed by quantitative real-time PCR (qRT-PCR) and $\mathrm{T}$ cells from the perigastric lymph nodes (GLNs) that were assayed by flow cytometry.

The Th1 response was detectable, but it was not particularly robust, and there were no significant differences in Ifng expression in gastric tissues from infected Egfriflfl,$E g f r^{\Delta m y e}$, or Lys $M^{\mathrm{Cre} / \mathrm{Cre}}$ mice (Supplemental Figure 20A). We observed a modest, but not statistically significant, induction of the $\mathrm{CD} 4^{+} \mathrm{IFN}-\gamma^{+}$cell population in the GLNs from infected Egfr flflfl $^{\text {mice }}$ (Supplemental Figure 20B). However, the $\mathrm{CD} 4^{+} \mathrm{IFN}-\gamma^{+}$cell population was significantly decreased in Egfr ${ }^{\text {mye }}$ GLNs (Supplemental Figure 20B). The lamina propria $\mathrm{T}$ cells showed a significant increase in both $\mathrm{T}$ box 21 (Tbx21, also known as Tbet), a transcription factor regulating Th1 differentiation, and Ifng expression in Egfrl/fl mice, but only Tbx21 expression was significantly decreased in Egfr ${ }^{\text {मmye }}$ mice (Supplemental Figure 20C).

Th17 responses were more robust and more dependent on macrophage EGFR signaling at 4 months p.i. Il17a mRNA levels were significantly increased in infected Egfrif/fl and $L y s M^{\mathrm{Cre} / \mathrm{Cre}}$ gastric tissues and decreased in infected Egfr ${ }^{4 m y e}$ gastric tissues (Figure 9A). Similarly, IL-17 protein levels were increased in infected Egfrfl/fl gastric tissues and significantly decreased in Egfr ${ }^{\Delta m y e}$ tissues (Figure 9B). Consistent with these data, the $\mathrm{CD} 4^{+} \mathrm{IL}-17^{+}$cell population in $E g \mathrm{fr}^{\mathrm{fl} / \mathrm{fl}} \mathrm{GLNs}$ was significantly increased with infection, and the $\mathrm{CD}^{+}{ }^{+} \mathrm{IL}-17^{+}$cell population in Egfrumye GLNs was significantly decreased (Figure 9C). Both RAR-related orphan receptor C (Rorc, also known as $R O R \gamma$ ), encoding for a transcription factor that mediates Th17 differentiation, and Il17a mRNA levels were very highly induced in $\mathrm{CD} 4^{+} \mathrm{T}$ cells from the lamina propria of Egfrl/fl mice (Figure 9D). Notably, Egfr ${ }^{\text {smye }}$ mice had significantly lower Rorc and Il17a expression levels in $\mathrm{CD}^{+} \mathrm{T}$ cells from the lamina propria (Figure 9D).

IL-23, composed of IL-12p19 and IL-12p40, and TGF- $\beta$ are necessary for Th17 differentiation (62). IL-12p40 protein (Supplemental Table 1) and Tgfb mRNA levels (Figure 5B) were significantly decreased in Egfr ${ }^{\Delta m y e}$ gastric tissue. Moreover, mRNA levels of macrophage-derived Il23a (Supplemental Figure 21) and Tgfb (Supplemental Figure 12B) were significantly reduced in Egfrsmye BMmacs. Taken together, these data indicate a diminished potential for Th17 differentiation. These data also indicate that EGFR signaling in macrophages is important for the Th17 response to H. pylori infection. The decreased Th17 response in Egfr ${ }^{\Delta m y e}$ mice likely contributes to the decreased histologic gastritis.

In contrast to the diminished Th17 response in Egfrumye mice, the Treg response was substantially increased. mRNA expression of Foxp3, a transcription factor specific to Tregs, was not induced in infected $E g \mathrm{fr}^{\mathrm{fl} / \mathrm{fl}}$ gastric tissues, but was significantly upregulated in Egfr ${ }^{\Delta m y e}$ tissues (Figure 10A). Similarly, the CD $4^{+} \mathrm{CD} 25^{+} \mathrm{FOXP3}{ }^{+}$ cell population in Egframe GLNs was significantly larger than in Egfriflfl GLNs (Figure 10B). Significantly enhanced Foxp3, Il1O, and $\mathrm{Tg} f b$ expression in $\mathrm{CD}^{+} \mathrm{T}$ cells from the lamina propria of Egfr ${ }^{\text {smye }}$ mice further confirmed the enhancement of the Treg population during infection (Figure 10C). These data implicate a potential role for macrophage EGFR signaling in the Treg response during H.pylori infection.

We also assessed the role of macrophage EGFR in the initiation of the adaptive immune response to $H$. pylori. We examined the Th1, Th17, and Treg populations at 2 months p.i. (Supplemental Figure 22). In contrast to the 4-month p.i. time point, there was no increase in Th1, Th17, or Treg populations with $H$. pylori infection in the Egfrlflfl or Egframye GLNs when assessed by flow cytometry (Supplemental Figure 22, A, C, and E). There were, however, modest increases in expression levels of the Th1- and Th17-related genes in infected Egfrfl/fl mice, levels that were decreased in Egfr ${ }^{\text {smye }}$ mice (Supplemental Figure 22, B and D). Together, these data suggest that the gastric $\mathrm{T}$ cell response is still developing at the 2-month p.i. time point and that macrophage-T cell interaction is best assessed at 4 months p.i. in the H.pylori model.

\section{Discussion}

EGFR regulates many epithelial cell functions, in addition to playing a procarcinogenic role in many cancers, including gastric cancer $(39,63-66)$. While EGFR signaling in macrophages has been demonstrated within the context of inflammation and cancer $(18,19)$, our work reveals the central role that EGFR has in regulating macrophage activation and function in response to bacterial pathogens. Gastric carcinogenesis derives from deleterious $H$. pylori-induced chronic inflammation that is substantially driven by macrophages $(28,31,33,34,49,55)$. We now show that EGFR signaling in macrophages has a causal role in gastric inflammation and is associated with $H$. pylori-induced gastric carcinogenesis. Specifically, human Gmacs have abundant levels of p-EGFR, in the setting of both gastritis and intestinal metaplasia, and this continues in gastric carcinoma. As such, 
macrophage EGFR is a potential therapeutic target for this and other inflammation-mediated diseases and cancers.

Myeloid-specific deletion of EGFR in mice significantly attenuated gastritis, with a commensurate increase in $H$. pylori burden. This decrease in gastric damage was marked by decreased proinflammatory cytokine and chemokine levels. Similarly, the diminished clinical severity of infectious colitis in Egfrumve mice, despite increased C. rodentium burden, further implicates the importance of EGFR signaling in macrophages in the deleterious inflammatory response to bacterial infections. This concept was also supported by the decrease in proinflammatory cytokine expression in colonic tissues from infected Egfrumye mice.

Either inhibition of signaling or genetic knockout of EGFR led to decreased M1 and Mreg activation during bacterial infection in vitro and in vivo. Canonical stimuli for M1, M2, and Mreg activation also resulted in decreased gene expression of subset-specific markers. Macrophage activation is critical for defining function, and our studies have established that EGFR assumes a central role in regulating this key step in macrophage biology. Importantly, we have used the term "activation" instead of "polarization," because this study is focused on gene expression patterns related specifically to macrophage activation. It should be noted, however, that we have assessed the expression of arginase 2 (Arg2) and ornitihine decarboxylase $(O d c)$, which encode for important enzymes involved in polyamine biosynthesis and which we have previously shown to be upregulated in $H$. pylori-infected macrophages $(33,67)$. In BMmacs with either chemical inhibition or knockout of EGFR, there was no affect on $H$. pylori-induced expression of $\mathrm{Arg} 2$ or $\mathrm{Odc}$ (data not shown), suggesting that EGFR signaling does not have global effects on all macrophage genes induced by $H$. pylori.

As M1 activation is responsible for proinflammatory, antimicrobial responses to bacterial pathogens, loss of M1 activation in Egfrumye mice is likely driving the decreased disease severity and increased bacterial load in the models used here. Additionally, EGFR signaling in macrophages has marked effects on adaptive immunity, as it was required for Th17 responses, which have been implicated in clearance of H.pylori (68) and C.rodentium (69) infection.

The link between EGFR and NF- $\kappa \mathrm{B}$ provides a mechanism for the observed decrease in cytokine production by macrophages. Inhibition or deletion of EGFR led to significantly decreased $\mathrm{NF}-\kappa \mathrm{B}$ pathway signaling, including the upstream components MyD88, p-IKBK, and p-NFKBIA, as well as decreased RELA nuclear translocation and NF- $\kappa \mathrm{B}$ activity. It should be noted that $\mathrm{MyD} 88^{-/-}$mice have been reported to exhibit attenuated gastritis and increased bacterial burden with $H$. pylori infection (70), consistent with our data herein regarding myeloid-specific deletion of Egfr. Thus, MyD88 may provide a link between EGFR signaling and NF- $\mathrm{KB}$ in macrophages.

We have also implicated MAPK1/3 signaling as an additional target of EGFR signaling, which appears to be important in modulating macrophage activation in response to H.pylori. Since many potential pathways may be influenced by EGFR signaling, determining additional downstream targets of EGFR activation in macrophages is currently being investigated in our laboratory through proteomic approaches. These studies will enhance our understanding of the mechanisms controlling macrophage responses.
Furthermore, phosphorylation of EGFR was detectable at S1046/47 as well as at the canonical Y1068, indicating that initiation of EGFR signaling in macrophages can be ligand independent $(42,43)$. We have implicated a potential role for TNF- $\alpha$ in such a ligand-independent mechanism. Future studies to determine the mechanism by which EGFR signaling is initiated in macrophages in response to bacterial infections are warranted. These studies could provide useful targets for therapeutic intervention in preventing EGFR activation in macrophages during chronic inflammation.

In sum, our work outlines a new role for EGFR signaling in regulating the macrophage-mediated host responses to bacterial infections. We have implicated EGFR as a central element in macrophage activation, with broad effects on immunopathogenesis. In the particular case of $H$. pylori infection, specific inhibition of pathways that activate macrophage EGFR signaling could prove a useful chemopreventive strategy in high-risk subjects, since antibiotic treatment of $H$. pylori once precancerous lesions of atrophic gastritis and intestinal metaplasia have occurred does not reduce gastric cancer risk $(71,72)$. Further, this work provides an impetus for further studies regarding the role of EGFR in macrophages, especially as it relates to the treatment and prevention of other inflammatory diseases and associated carcinogenesis.

\section{Methods}

Materials. All reagents used for cell culture were from Invitrogen. Reagents for RNA extraction were from QIAGEN. Reagents for cDNA synthesis and qRT-PCR were purchased from Bio-Rad. Gefitinib [ $N$-(3-chloro-4-fluorophenyl)-7-methoxy-6-(3-morpholinopropoxy) quinazolin-4-amine] was obtained from Selleck Chemicals. AG1478 [ $\mathrm{N}$-(3-chlorophenyl)-6,7-dimethoxyquinazolin-4-amine] was obtained from Cayman Chemical. PD153035 [N-(3-bromophenyl)-6,7-dimethoxyquinazoline] was obtained from EMD Millipore. The following compounds were obtained from EMD Millipore: PD153035 [ $N$-(3-bromophenyl)6,7-dimethoxyquinazoline] and ERKi [3-(2-aminoethyl)-5-((4-ethoxyphenyl)methylene)-2,4-thiazolidinedione, $\mathrm{HCl}]$.

Abs. See Supplemental Table 3 for information regarding all Abs used in this study.

Bacteria, cells, culture conditions, and infections. H. pylori PMSS1, SS1, and 7.13 were grown and used as previously described $(32,33,49)$.

The murine macrophage cell line RAW 264.7 was maintained in complete DMEM, supplemented with 10\% FBS, 2 mM L-glutamine, $100 \mathrm{U} / \mathrm{ml}$ penicillin, $100 \mu \mathrm{g} / \mathrm{ml}$ streptomycin, $50 \mathrm{mg} / \mathrm{ml}$ gentamicin, $25 \mathrm{mM}$ HEPES, and $10 \mathrm{mM}$ sodium pyruvate. The human monocytic cell line THP-1 was maintained in complete DMEM, supplemented with $10 \%$ FBS, $2 \mathrm{mM}$ L-glutamine, $100 \mathrm{U} / \mathrm{ml}$ penicillin, $100 \mu \mathrm{g} / \mathrm{ml}$ streptomycin, and $50 \mathrm{mg} / \mathrm{ml}$ gentamicin. THP-1 cells were differentiated into adherent macrophages with $10 \mathrm{ng} / \mathrm{ml}$ PMA for 24 hours. Cells were then washed and incubated in complete DMEM without PMA for 24 hours prior to experimentation. RAW 264.7 cells and THP-1 cells were acquired from ATCC. ATCC authenticates all cell lines before distribution and also tests for and confirms that all cell lines are free of mycoplasma contamination. The murine immortalized BMmac, NF- $\mathrm{BB}$ luciferase reporter cell line, NGL, was provided by Timothy Blackwell (Vanderbilt University Medical Center) $(58,59)$ and was maintained in complete DMEM, supplemented with 10\% FBS, 2 mM L-glutamine, $100 \mathrm{U} / \mathrm{ml}$ penicillin, $100 \mu \mathrm{g} / \mathrm{ml}$ streptomycin, and $50 \mathrm{mg} / \mathrm{ml}$ genta- 
micin. None of the cell lines used in this study is found in the database of commonly misidentified cells lines maintained by the International Cell Line Authentication Committee.

BMmacs were isolated from all mouse genotypes used in this study as previously described (73), with the following exceptions: rbc were lysed with ammonium-chloride-potassium lysing buffer for 4 minutes prior to plating BM cells. Recombinant macrophage CSF (M-CSF) was used at a concentration of $20 \mathrm{ng} / \mathrm{ml}$ for 7 days. BMDCs were isolated from all mouse genotypes as previously described (74). Recombinant granulocyte-monocyte CSF (GM-CSF) was used at a concentration of $40 \mathrm{ng} / \mathrm{ml}$ for 9 days. M-CSF and GM-CSF were purchased from PeproTech.

All cells were incubated in serum-free media for 14 to 16 hours prior to infection for assays measuring p-EGFR. All pharmacological inhibitors were added 1 hour before infection. Cells were infected at an MOI of 100 for $H$. pylori experiments. Media without antibiotics were used for all experiments with live bacteria.

Animal studies. Egfr ${ }^{\text {mmye }}$ and Egfrt/fl villin 1-Cre (Vill $\left.{ }^{\mathrm{Cre} /+}\right)$ mice were provided by Fang Yan (Vanderbilt University Medical Center). Egfrfl/fl mice were generated by backcrossing Egfrlf/fl Vill $^{\text {Cre/+ }}$ mice with WT mice to remove the Cre allele. $L y s M^{\mathrm{Cr} / \mathrm{Cre}}$ mice were purchased from The Jackson Laboratory. Excision of the Egfr alleles was confirmed by PCR (See Supplemental Table 2 for primer information). Six- to twelveweek-old male mice were exclusively used for these studies, regardless of genotype, and mice were not removed from the cages in which they were weaned. Male mice were selected for this study, because female mice have been shown to be protected from gastric damage in experimental $H$. pylori infection (75). Sample sizes were based on previous studies from our laboratory $(32,33,55)$ and mice were randomly selected for control and experimental groups, provided they met the sex and age requirements. Mice were infected orogastrically with $5 \times$ $10^{8} \mathrm{CFU}$ H. pylori SS1 or PMSS1. Inoculations occurred once for Gmac studies and 3 times, every other day, for all other H.pylori studies. Animals were sacrificed 48 hours p.i. for Gmacs and 1 month, 2 months, or 4 months p.i. for all other $H$. pylori studies. Colonization was assessed by serial dilution and culture. Histology was assessed in a completely blinded manner by a gastrointestinal pathologist, M. Blanca Piazuelo, using the updated Sydney System (55).

Human tissues. Human gastric tissue samples were obtained as previously described $(39,41)$. Both a Vanderbilt University Medical Center TMA (39) and samples from Colombia (41) were used for this study.

qRT-PCR. RNA was isolated from cells and tissues using RNeasy kits. cDNA was prepared from each sample using $1 \mu \mathrm{g}$ RNA and the iScript cDNA synthesis kit. PCR was performed as previously described (55). Primers are listed in Supplemental Table 2.

Western blot analysis. Cells were lysed in CellLytic MT Reagent (Sigma-Aldrich) or using the NE-PER Protein Extraction Kit (Pierce Biotechnology, Thermo Fisher Scientific). All lysis buffers were supplemented with the Protease Inhibitor Cocktail (Set III; Calbiochem) and the Phosphatase Inhibitor Cocktail (Set I; Calbiochem). Protein concentration was determined using the BCA protein assay (Pierce Biotechnology, Thermo Fisher Scientific). Primary and secondary Abs are listed in Supplemental Table 3. Densitometry was performed using ImageStudio, version 4.0.21 (LI-COR).

Measurement of NO. The concentration of the oxidized metabolite of NO nitrite $\left(\mathrm{NO}_{2}^{-}\right)$was assessed by the Griess reaction as previously described (32).
$N F-\kappa B$ reporter assay. See Supplemental Methods for detailed methodology.

Luminex assay. See Supplemental Methods for detailed methodology. Immunofluorescence staining for CD68, p-EGFR, $t$-EGFR, and cleaved caspase 3. Immunofluorescence staining was performed on BMmacs after $H$. pylori infection, on murine gastric tissues from mice 4 months after infection with $H$. pylori SS1, and on human gastric tissues from Colombia and the Vanderbilt University TMA, as previously published $(39,55)$. Antigen retrieval was performed as previously described (39). (See Supplemental Table 3 for Ab information). Slides were imaged using a SPOT RT slider camera system (Diagnostic Instruments Inc.) on a Nikon E800 microscope. Images were all modestly adjusted in Image (NIH) with the brightness and contrast tool.

Quantification of human TMA immunofluorescence. See Supplemental Methods for detailed methodology.

Purification of Gmacs. Gmacs were purified as previously described (33).

T cell studies. See Supplemental Methods for detailed methodology.

Flow cytometry. Flow cytometry was performed as previously described (33). See additional details in Supplemental Methods. All information regarding Abs is available in Supplemental Table 3.

Statistics. All data shown represent the mean \pm SEM. At least 3 biological replicates were performed for all studies using cell cultures. Where data was normally distributed, a 2-tailed Student's $t$ test was used to determine significance in experiments with only 2 groups, and 1-way ANOVA with the Newman-Keuls test was used to determine significant differences between multiple test groups. In cases in which data were not normally distributed, a 1-way ANOVA with a Kruskal-Wallis post test, followed by a Mann-Whitney $U$ test, were performed. All statistics were performed using GraphPad Prism 5.0 (GraphPad Software). A P value of less than 0.05 was considered significant.

Study approval. Animals were used under protocol M/10/155, which was approved by the IACUC of Vanderbilt University. WT C57BL/6 mice were bred and maintained under IACUC protocol V/13/230. Human tissues were obtained from a Vanderbilt University Medical Center repository, and nonhuman exemptions were granted by the IRB of Vanderbilt University, as all information was completely deidentified.

\section{Author contributions}

$\mathrm{DMH}$ designed and performed experiments, analyzed data, interpreted results, and prepared the manuscript. KS, MA, TGV, DOV, and MMA performed experiments. DPB bred and maintained the mice. MKW generated the TMA and associated clinical information. MBP performed all histopathological scoring. RMP provided funding support and critical review of the manuscript. KTW obtained funding, designed experiments, interpreted results, and prepared the manuscript.

\section{Acknowledgments}

We thank Frank Revetta and Joseph Roland (Vanderbilt University) for their assistance with the human TMA and Alberto Delgado (Vanderbilt University) for assistance with slide preparation for immunofluorescence and immunohistochemistry. This work was funded by NIH grants R01DK053620, R01AT004821, R01CA190612, P01CA116087, and P01CA028842 (to KTW); a Department of Veterans Affairs Merit Review grant I01BX001453 
(to KTW); the Thomas F. Frist Sr. Endowment (to KTW); and the Vanderbilt Center for Mucosal Inflammation and Cancer (to KTW). The use of the human TMA was supported by the Vanderbilt Digestive Disease Research Center, funded by the NIH grant P3ODK058404, and the Specialized Program of Research Excellence (SPORE) in GI Cancer, funded by the NIH grant P50CA095103. DMH was supported by NIH grants,
T32GM008554 and F31DK10715. Flow cytometry was supported by NIH grants P3ODK058404 and P30CA068485.

Address correspondence to: Keith T. Wilson, Vanderbilt University School of Medicine, 2215 Garland Avenue, 1030C Medical Research Building IV, Nashville, Tennessee 37232, USA. Phone: 615.343.5675; E-mail: keith.wilson@vanderbilt.edu.
1. Benoit M, Desnues B, Mege JL. Macrophage polarization in bacterial infections. JImmunol. 2008;181(6):3733-3739.

2. Mosser DM, Edwards JP. Exploring the full spectrum of macrophage activation. Nat Rev Immunol. 2008;8(12):958-969.

3. Murray PJ, Wynn TA. Protective and pathogenic functions of macrophage subsets. Nat Rev Immunol. 2011;11(11):723-737.

4. Mosser DM. The many faces of macrophage activation. J Leukoc Biol. 2003;73(2):209-212.

5. Martinez FO, Gordon S. The M1 and M2 paradigm of macrophage activation: time for reassessment. F100OPrime Rep. 2014;6:13.

6. Anderson CF, Mosser DM. A novel phenotype for an activated macrophage: the type 2 activated macrophage. JLeukoc Biol. 2002;72(1):101-106.

7. Ostuni R, Kratochvill F, Murray PJ, Natoli G. Macrophages and cancer: from mechanisms to therapeutic implications. Trends Immunol. 2015;36(4):229-239.

8. Pander J, et al. Activation of tumor-promoting type 2 macrophages by EGFR-targeting antibody cetuximab. Clin Cancer Res. 2011;17(17):5668-5673.

9. Fleming BD, Mosser DM. Regulatory macrophages: setting the threshold for therapy. Eur J Immunol. 2011;41(9):2498-2502.

10. Ruffell D, et al. A CREB-C/EBPbeta cascade induces M2 macrophage-specific gene expression and promotes muscle injury repair. Proc Natl Acad Sci USA. 2009;106(41):17475-17480.

11. Biswas SK, et al. A distinct and unique transcriptional program expressed by tumorassociated macrophages (defective NF-kappaB and enhanced IRF-3/STAT1 activation). Blood. 2006;107(5):2112-2122.

12. Foster SL, Hargreaves DC, Medzhitov R. Gene-specific control of inflammation by TLR-induced chromatin modifications. Nature. 2007;447(7147):972-978.

13. Ghisletti S, et al. Identification and characterization of enhancers controlling the inflammatory gene expression program in macrophages. Immunity. 2010;32(3):317-328.

14. Krausgruber T, et al. IRF5 promotes inflammatory macrophage polarization and TH1-TH17 responses. Nat Immunol. 2011;12(3):231-238.

15. Lawrence T, Natoli G. Transcriptional regulation of macrophage polarization: enabling diversity with identity. Nat Rev Immunol. 2011;11(11):750-761.

16. Tietzel I, Mosser DM. The modulation of macrophage activation by tyrosine phosphorylation. Front Biosci. 2002;7:d1494-d1502.

17. Liu B, Neufeld AH. Activation of epidermal growth factor receptor signals induction of nitric oxide synthase- 2 in human optic nerve head astrocytes in glaucomatous optic neuropathy. Neurobiol Dis. 2003;13(2):109-123.
18. Lanaya H, et al. EGFR has a tumour-promoting role in liver macrophages during hepatocellular carcinoma formation. Nat Cell Biol. 2014;16(10):972-981, 1.

19. Lu N, et al. Activation of the epidermal growth factor receptor in macrophages regulates cytokine production and experimental colitis. J Immunol. 2014;192(3):1013-1023.

20. Scholes AG, Hagan S, Hiscott P, Damato BE, Grierson I. Overexpression of epidermal growth factor receptor restricted to macrophages in uveal melanoma. Arch Ophthalmol. 2001;119(3):373-377.

21. Cover TL, Blaser MJ. Helicobacter pylori in health and disease. Gastroenterology. 2009;136(6):1863-1873.

22. [No authors listed]. Infection with Helicobacter pylori. IARC Monogr Eval Carcinog Risks Hum. 1994;61:177-240.

23. Sibony M, Jones NL. Recent advances in Helicobacter pylori pathogenesis. Curr Opin Gastroenterol. 2012;28(1):30-35.

24. Nomura A, Stemmermann GN, Chyou PH, Kato I, Perez-Perez GI, Blaser MJ. Helicobacter pylori infection and gastric carcinoma among Japanese Americans in Hawaii. N Engl J Med. 1991;325(16):1132-1136.

25. Parsonnet J, et al. Helicobacter pylori infection and the risk of gastric carcinoma. N Engl JMed. 1991;325(16):1127-1131.

26. Peek RM, Blaser MJ. Helicobacter pylori and gastrointestinal tract adenocarcinomas. Nat Rev Cancer. 2002;2(1):28-37.

27. Chaturvedi R, et al. Increased Helicobacter pylori-associated gastric cancer risk in the Andean region of Colombia is mediated by spermine oxidase. Oncogene. 2015;34(26):3429-3440.

28. Hardbower DM, de Sablet T, Chaturvedi R, Wilson KT. Chronic inflammation and oxidative stress: the smoking gun for Helicobacter pylori-induced gastric cancer?. Gut Microbes. 2013;4(6):475-481.

29. Hardbower DM, Peek RM, Wilson KT. At the Bench: Helicobacter pylori, dysregulated host responses, DNA damage, and gastric cancer. JLeukoc Biol. 2014;96(2):201-212.

30. Peek RM, Fiske C, Wilson KT. Role of innate immunity in Helicobacter pylori-induced gastric malignancy. Physiol Rev. 2010;90(3):831-858.

31. Robinson K, Argent RH, Atherton JC. The inflammatory and immune response to Helicobacter pylori infection. Best Pract Res Clin Gastroenterol. 2007;21(2):237-259.

32. Chaturvedi R, et al. Polyamines Impair Immunity to Helicobacter pylori by Inhibiting L-Arginine Uptake Required for Nitric Oxide Production. Gastroenterology. 2010;139(5):1686-1698,1698.e1.

33. Lewis ND, et al. Immune evasion by Helicobacter pylori is mediated by induction of macrophage arginase II. J Immunol. 2011;186(6):3632-3641.

34. Kaparakis M, et al. Macrophages are mediators of gastritis in acute Helicobacter pylori infection in C57BL/6 mice. Infect Immun. 2008;76(5):2235-2239.

35. Mera R, et al. Long term follow up of patients treated for Helicobacter pylori infection. Gut. 2005;54(11):1536-1540.

36. Kelly M, et al. Essential role of the type III secretion system effector NleB in colonization of mice by Citrobacter rodentium. Infect Immun. 2006;74(4):2328-2337.

37. Van Kaer L, et al. CD $8 \alpha \alpha(+)$ innate-type lymphocytes in the intestinal epithelium mediate mucosal immunity. Immunity. 2014;41(3):451-464.

38. Schreiber HA, et al. Intestinal monocytes and macrophages are required for $\mathrm{T}$ cell polarization in response to Citrobacter rodentium. J Exp Med. 2013;210(10):2025-2039.

39. Chaturvedi R, et al. Activation of EGFR and ERBB2 by Helicobacter pylori results in survival of gastric epithelial cells with DNA damage. Gas troenterology. 2014;146(7):1739-51.e14.

40. de Sablet T, et al. Phylogeographic origin of Helicobacter pylori is a determinant of gastric cancer risk. Gut. 2011;60(9):1189-1195.

41. Gobert AP, et al. Disruption of nitric oxide signaling by Helicobacter pylori results in enhanced inflammation by inhibition of heme oxygenase-1. JImmunol. 2011;187(10):5370-5379.

42. Nishimura M, et al. TAK1-mediated serine/threonine phosphorylation of epidermal growth factor receptor via p38/extracellular signal-regulated kinase: NF-\{kappa\}B-independent survival pathways in tumor necrosis factor alpha signaling. Mol Cell Biol. 2009;29(20):5529-5539.

43. Singhirunnusorn P, Ueno Y, Matsuo M, Suzuki S, Saiki I, Sakurai H. Transient suppression of ligand-mediated activation of epidermal growth factor receptor by tumor necrosis factor-alpha through the TAK1-p38 signaling pathway. J Biol Chem. 2007;282(17):12698-12706.

44. Marikovsky M, et al. Appearance of heparinbinding EGF-like growth factor in wound fluid as a response to injury. Proc Natl Acad Sci USA. 1993;90(9):3889-3893.

45. Hobbs SS, et al. TNF transactivation of EGFR stimulates cytoprotective COX-2 expression in gastrointestinal epithelial cells. Am J Physiol Gas trointest Liver Physiol. 2011;301(2):G220-G229.

46. Lee TC, Threadgill DW. Generation and validation of mice carrying a conditional allele of the epidermal growth factor receptor. Genesis. 2009;47(2):85-92.

47. Algood HM, Gallo-Romero J, Wilson KT, Peek RM, Cover TL. Host response to Helicobacter pylori infection before initiation of the adaptive immune response. FEMS Immunol Med Microbiol. 
2007;51(3):577-586.

48. Asim M, et al. Helicobacter pylori induces ERK-dependent formation of a phosphoc-Fos c-Jun activator protein-1 complex that causes apoptosis in macrophages. J Biol Chem. 2010;285(26):20343-20357.

49. Gobert AP, et al. Helicobacter pylori induces macrophage apoptosis by activation of arginase II. J Immunol. 2002;168(9):4692-4700.

50. Israel DA, Peek RM. The role of persistence in Helicobacter pylori pathogenesis. Curr Opin Gastroenterol. 2006;22(1):3-7.

51. Noto JM, et al. Iron deficiency accelerates Helicobacter pylori-induced carcinogenesis in rodents and humans. J Clin Invest. 2013;123(1):479-492.

52. Gobert AP, et al. Protective role of arginase in a mouse model of colitis. JImmunol. 2004;173(3):2109-2117.

53. Chaturvedi R, et al. L-arginine availability regulates inducible nitric oxide synthase-dependent host defense against Helicobacter pylori. Infect Immun. 2007;75(9):4305-4315.

54. Lewis ND, et al. Arginase II restricts host defense to Helicobacter pylori by attenuating inducible nitric oxide synthase translation in macrophages. J Immunol. 2010;184(5):2572-2582.

55 . Gobert AP, et al. Heme oxygenase-1 dysregulates macrophage polarization and the immune response to Helicobacter pylori. JImmunol. 2014;193(6):3013-3022.

56. Backert S, Naumann M. What a disorder: proinflammatory signaling pathways induced by Helicobacter pylori. Trends Microbiol. 2010;18(11):479-486.

57. Brandt S, Kwok T, Hartig R, König W, Backert
S. NF-kappaB activation and potentiation of proinflammatory responses by the Helicobacter pylori CagA protein. Proc Natl Acad Sci USA. 2005;102(26):9300-9305.

58. Everhart MB, et al. Duration and intensity of NF-kappaB activity determine the severity of endotoxin-induced acute lung injury. J Immunol. 2006;176(8):4995-5005.

59. Han W, et al. NADPH oxidase limits lipopolysaccharide-induced lung inflammation and injury in mice through reduction-oxidation regulation of NF-אB activity. JImmunol. 2013;190(9):4786-4794.

60. Orton RJ, Sturm OE, Vyshemirsky V, Calder M, Gilbert DR, Kolch W. Computational modelling of the receptor-tyrosine-kinase-activated MAPK pathway. Biochem J. 2005;392(Pt 2):249-261.

61. Schulze WX, Deng L, Mann M. Phosphotyrosine interactome of the ErbB-receptor kinase family. Mol Syst Biol. 2005;1:2005.0008.

62. Dienz O, Rincon M. The effects of IL-6 on CD4 T cell responses. Clin Immunol. 2009;130(1):27-33.

63. Nicholson RI, Gee JM, Harper ME. EGFR and cancer prognosis. Eur J Cancer. 2001;37 Suppl 4:S9-15.

64. Scaltriti M, Baselga J. The epidermal growth factor receptor pathway: a model for targeted therapy. Clin Cancer Res. 2006;12(18):5268-5272.

65. Tokunaga A, et al. Clinical significance of epidermal growth factor (EGF), EGF receptor, and c-erbB-2 in human gastric cancer. Cancer. 1995;75(6 Suppl):1418-1425.

66. Yan F, et al. Epidermal growth factor receptor activation protects gastric epithelial cells from Helicobacter pylori-induced apoptosis. Gastroenterology. 2009;136(4):1297-1307, e1.

67. Cheng Y, et al. Helicobacter pylori-induced macrophage apoptosis requires activation of ornithine decarboxylase by c-Myc. J Biol Chem. 2005;280(23):22492-22496.

68. DeLyria ES, Redline RW, Blanchard TG. Vaccination of mice against $\mathrm{H}$ pylori induces a strong Th-17 response and immunity that is neutrophil dependent. Gastroenterology. 2009;136(1):247-256.

69. Mangan PR, et al. Transforming growth factorbeta induces development of the $\mathrm{T}(\mathrm{H}) 17$ lineage. Nature. 2006;441(7090):231-234.

70. $\mathrm{Rad} \mathrm{R}$, et al. Toll-like receptor-dependent activation of antigen-presenting cells affects adaptive immunity to Helicobacter pylori. Gastroenterology. 2007;133(1):150-163.e3.

71. Dalal RS, Moss SF. At the bedside: Helicobacter pylori, dysregulated host responses, DNA damage, and gastric cancer. J Leukoc Biol. 2014;96(2):213-224.

72. Ford AC, Forman D, Hunt RH, Yuan Y, Moayyedi P. Helicobacter pylori eradication therapy to prevent gastric cancer in healthy asymptomatic infected individuals: systematic review and meta-analysis of randomised controlled trials. BMJ. 2014;348:g3174.

73. Weischenfeldt J, Porse B. Bone Marrow-Derived Macrophages (BMM): Isolation and Applications. CSH Protoc. 2008;2008:pdb.prot5080.

74. Matheu MP, Sen D, Cahalan MD, Parker I. Generation of bone marrow derived murine dendritic cells for use in 2-photon imaging. J Vis Exp. 2008;(17):pii: 773.

75. Sheh A, et al. $17 \beta$-estradiol and tamoxifen prevent gastric cancer by modulating leukocyte recruitment and oncogenic pathways in Helicobacter pylori-infected INS-GAS male mice. Cancer Prev Res (Phila). 2011;4(9):1426-1435. 